\title{
1 C. elegans RAP-1 reinforces LET-60/Ras induction of cell fate
}

2 Neal R. Rasmussen *, Daniel J. Dickinson ${ }^{\dagger}$, David J. Reiner * $\neq$

$3{ }^{*}$ Center for Translational Cancer Research, Institute of Biosciences and Technology, Texas

4 A\&M Health Science Center, Houston, TX, 77030, USA, ${ }^{\dagger}$ Department of Molecular

5 Biosciences, University of Texas, Austin, TX, 78705, ${ }^{\ddagger}$ Department of Medical Physiology,

6 College of Medicine, Texas A\&M University, College Station, TX 77843, USA. 


\section{$7 \quad$ RAP-1 reinforces LET-60 signaling}

8 Key Words: Ras, Rap1, Raf, PDZ-GEF, CRISPR

9 Correspondence: Center for Translational Cancer Research

10

Institute of Biosciences and Technology

11

Texas A\&M Health Science Center

12

2121 W. Holcombe Blvd.

13

Houston, TX 77030

14

(713) 677-7416

15

dreiner@ibt.tamhsc.edu 


\section{Abstract}

17 The notoriety of the small GTPase Ras as the most mutated oncoprotein has led to a well-

18 characterized signaling network largely conserved across metazoans. Yet the role of its close

19 relative Rap1 (Ras Proximal), which shares 100\% identity between their core effector binding

20 sequences, remains unclear. A long-standing controversy in the field is whether Rap1 also

21 functions to activate the canonical Ras effector, the S/T kinase Raf. We used the developmentally

22 simpler Caenorhabditis elegans, which lacks the extensive paralog redundancy of vertebrates, to

23 examine the role of RAP-1 in two distinct LET-60/Ras-dependent cell fate patterning events:

24 induction of $1^{\circ}$ vulval precursor cell (VPC) fate and of the excretory duct cell. Fluorescently tagged

25 endogenous RAP-1 is localized to plasma membranes and is expressed ubiquitously, with even

26 expression levels across the VPCs. RAP-1 and its activating GEF PXF-1 function cell

27 autonomously and are necessary for maximal induction of $1^{\circ}$ VPCs. Critically, mutationally

28 activated endogenous RAP-1 is sufficient both to induce ectopic $1^{\circ} \mathrm{s}$ and duplicate excretory duct

29 cells. Like endogenous RAP-1, before induction GFP expression from the pxf-1 promoter is

30 uniform across VPCs. However, unlike endogenous RAP-1, after induction GFP expression is

31 increased in presumptive $1^{\circ} \mathrm{s}$ and decreased in presumptive $2^{\circ} \mathrm{s}$. We conclude that RAP-1 is a

32 positive regulator that promotes Ras-dependent inductive fate decisions. We hypothesize that

33 PXF-1 activation of RAP-1 serves as a minor parallel input into the major LET-60/Ras signal

34 through LIN-45/Raf. 


\section{Introduction}

Ras, the founder of the Ras superfamily of small GTPases, is the most mutated oncoprotein in cancer (COSMIC v84; http://cancer.sanger.ac.uk/cosmic; (Papke and Der 2017). With diverse

38 functions throughout cell biology, members of the Ras superfamily are conserved across Metazoa.

39 Within given subfamilies, the core effector binding sequences are typically identical among $C$.

40 elegans, Drosophila, and mammals, suggesting functional conservation of GTPase interactions

41 with downstream effectors (Reiner and Lundquist 2016), many of which are also conserved across

42 Metazoa.

43 Small GTPases are typically membrane-bound through lipid prenylation and processing of 44 their C-termini (Hancock et al. 1990; Prior and Hancock 2012; Reiner and Lundquist 2016). They

45 function as molecular switches, cycling between GTP-bound (active) and GDP-bound (inactive)

46 states. Thus, activity of small GTPases is controlled by GEFs (guanine nucleotide exchange

47 factors), which displace GDP to allow GTP loading, and GAPs (GTPase activating proteins),

48 which stimulate the otherwise inefficient intrinsic GTPase activity that hydrolyzes GTP to GDP

49 (Wennerberg et al. 2005).

50 The Rap (as proximal) subfamily comprises GTPases closely related to Ras itself. Rap1

51 (previously known as K-Rev1) shares identical core effector binding sequences with Ras, while

52 Rap2 diverges at one residue in the core effector binding sequence (Fig S1, 2). The Rap subfamily

53 shares some GEFs and GAPs with the Ras subfamily, but also has some GEFs and GAPs that are

54 specific to Raps (Raaijmakers and Bos 2009; Gloerich and Bos 2011). Historically, Rap1 has

55 mostly been implicated in the regulation of cell-cell junctions, which is generally not considered

56 to be a functional site of Ras action (Caron et al. 2000; Reedquist et al. 2000; Knox and Brown

57 2002; Bos 2005). Yet because of their identical core effector binding sequences, it has long been 
58 hypothesized that Ras and Rap1 share effectors and may interact in signaling networks, while this

59 has not been suggested for Rap2. Unfortunately, an early experimental artifact in cell culture

60 experiments clouded the role of Rap1 relative to Ras and its interaction with the canonical Ras

61 effector, the Raf Ser/Thr kinase. Activated Rap1 transfected into cells inhibited oncogenic

62 transformation and blocked activation of the Ras-Raf-MEK-ERK canonical MAP kinase cascade

63 (Kitayama et al. 1989; Kitayama et al. 1990; Sakoda et al. 1992; Cook et al. 1993), yet failed to

64 interfere with ERK activation in more physiologically relevant conditions (Zwartkruis et al. 1998).

65 This observation led to the probably erroneous model that Rap1 was a competitive inhibitor of

66 Ras. Because this observation was only observed under conditions of high ectopic expression

67 (Kitayama et al. 1989), a plausible explanation is that over-expressed activated Rap1 sequestered

68 Raf to cell-cell junctions where Ras-Raf signaling is typically not functional, thus diminishing

69 activation of Raf-MEK-ERK signal by endogenous Ras. Yet the role of Rap1 relative to Ras-Raf

70 signaling has remained murky since, despite certain conditions in which Rap1 appears to

71 contribute to Raf activation in cultured mammalian cells (York et al. 1998).

72 However, several lines of evidence support Rap1 contributing to tumorigenic growth through

73 activation of Raf, suggesting that Ras and Rap1 may function in parallel to activate Raf. Rap1 can

74 oncogenically transform mammalian tissue culture cells (Altschuler and Ribeiro-Neto 1998). Non-

75 canonical putative activating mutations in Rap1 have also been associated with Kabuki Syndrome

76 (Bogershausen et al. 2015), part of the RASopathy spectrum of heritable disorders associated with

77 inappropriate weak activation of the Ras-Raf signaling axis (Aoki et al. 2016). Though typically

78 not mutated itself as an oncogene, rare mutations in Rap1 have been observed in various cancers

79 (COSMIC v84; http://cancer.sanger.ac.uk/cosmic; Gyan et al. 2005). The paucity of such

80 activating mutations may be because of the strong role of Rap1 in assembly and maintenance of 
81 cell-cell junctions, which may counter tumorigenic growth if not spatially controlled. A

82 comparison to Rap1 may be drawn with the Rho GTPase family member, Rac. Oncogenic Rac

83 mutations are rare and are mostly found in melanoma (Sergent 1990; Krauthammer et al. 2012).

84 Yet while mostly not mutated to drive cancer, probably because of its central role in control of the

85 cytoskeleton, cell morphogenesis, and migration, inappropriate upstream activation of Rac can still

86 contribute to tumorigenesis (Lindsay et al. 2011; Srijakotre et al. 2017). This loss of regulation

87 versus mutational activation has also been observed with Rap1, with the loss of a number of

88 RapGAPs, implicating them as tumor suppressors (McLaughlin et al. 2013; Maertens and

89 Cichowski 2014; Zhao et al. 2015).

90 Studies in Drosophila suggest that Rap1 is necessary for maximal induction of the Raf-MEK-

91 ERK MAP kinase cascade, perhaps in parallel to Ras activation (Mishra et al. 2005; Mavromatakis

92 and Tomlinson 2012). Yet in Drosophila, too, results with Rap1 have been contradictory (Baril et

93 al. 2014). The Drosophila Roughened mutation, originally thought to be a gain-of-function

94 mutation, was subsequently inferred to be a dominant-negative mutation (Hariharan et al. 1991;

95 Mavromatakis and Tomlinson 2012). That Drosophila Rap1 is an essential gene further

96 complicates investigation of its in vivo function with regard to activation of Raf during

97 development. Consequently, there is great benefit to studying the role of Rap1 in a simple

98 developmental system where Rap1 is not required for viability, and where there is less concern

99 about genetic redundancy. Thus, we are investigating the role of Rap1 signaling in C. elegans,

100 where Rap1 loss-of-function mutants are viable, and there is no redundancy in gene subfamilies

101 compared to mammals (mammals possess three Ras-, two Rap1-, three Rap2-, and three Raf-

102 encoding genes). 
C. elegans encodes RAP-1 and RAP-2, which correspond to mammalian Rap1 and Rap2, respectively (C. elegans RAP-3, which has a non-conservative change in a critical residue in the core effector binding sequence, is likely to be a nematode-specific, functionally divergent Rap;

106 Fig. S1; Reiner and Lundquist 2016). Consistent with their roles in promoting cell-cell junctions 107 in Drosophila and mammalian cells, C. elegans RAP-1 and RAP-2 are redundant for larval molting 108 and epithelial integrity, the double mutant causing lethality, a phenotype that is echoed by the 109 deletion of the RapGEF, PXF-1 (Pellis-van Berkel et al. 2005). For assembly of cadherin-based 110 cell-cell junctions in C. elegans, RAP-1 functions redundantly with the Ras family small GTPase, 111 RAL-1 (Ras like; Frische et al. 2007). Yet thus far there has been no examination of the role of 112 RAP-1 in developmental patterning of the C. elegans vulval precursor cell (VPC) fates, a system 113 where the sole $C$. elegans Ras ortholog, LET-60, plays a central role. Therefore, we investigated 114 the role of RAP-1 in VPC fate patterning.

115 The six equipotent VPCs (P3-8.p) are induced by EGF secreted by the anchor cell (AC) in the 116 somatic gonad, such that the VPC nearest the AC (typically P6.p) is induced to assume $1^{\circ}$ fate 117 (Fig. 1; Sternberg 2005). LET-23/EGFR receives the EGF signal, and the $1^{\circ}$-promoting signal is 118 transduced by a canonical Ras-Raf-MEK-ERK (C. elegans LET-60-LIN-45-MEK-2-MPK-1) 119 MAP kinase cascade: this signal is necessary and sufficient for induction of $1^{\circ}$ fate (Sundaram 120 2013). Induced presumptive $1^{\circ}$ cells express DSL ligands (Chen and Greenwald 2004), which 121 signal neighboring VPCs via the LIN-12/Notch receptor to assume $2^{\circ}$ fate. LIN-12/Notch is 122 necessary and sufficient for $2^{\circ}$ fate (Greenwald et al. 1983; Greenwald and Kovall 2013).

123 Development of the wild-type $3^{\circ}-3^{\circ}-2^{\circ}-1^{\circ}-2^{\circ}-3^{\circ}$ pattern of VPC fates occurs with $99.8 \%$ 124 accuracy (Braendle and Felix 2008). During VPC fate patterning, cells are initially specified, then 125 become committed to their fate (Sternberg 2005). During this process, the VPC signaling network 
126 is at least partially re-programmed, perhaps contributing to final commitment and fidelity. For 127 example, after initial induction, LIN-12/Notch is internalized and degraded in presumptive $1^{\circ}$ cells

128 (Shaye and Greenwald 2002, 2005), thus precluding $2^{\circ}$-promoting signal in a cell that is specified

129 to $1^{\circ}$ fate. Conversely, presumptive $2^{\circ}$ cells express the LIN-12/Notch transcriptional client, LIP-

130 1/ERK phosphatase (Berset et al. 2001; Yoo et al. 2004), thereby prohibiting $1^{\circ}$-promoting

131 signaling in cells that are specified as $2^{\circ}$. Additionally, expression of a suite of other LIN-12/Notch

132 transcriptional client genes is altered after initial induction (Berset et al. 2001; Yoo et al. 2004;

133 Berset et al. 2005; Yoo and Greenwald 2005; Zhang and Greenwald 2011), supporting the idea of

134 network reprogramming. We observed further evidence consistent with VPC re-programming with

135 ral-1 promoter expression - initially expressed in all VPCs - subsequently being excluded from

136 presumptive $1^{\circ}$ cells, thereby prohibiting $2^{\circ}$-promoting signaling in from cells that are specified as

$137 \quad 1^{\circ}$ (Reiner 2011; Zand et al. 2011). Most of this re-programming at the transcriptional level occurs

138 prior to the first VPC division. Though we do not know the precise point at which terminal

139 commitment occurs (Wang and Sternberg 1999), we hypothesize that transcriptional and

140 potentially post-translational network re-programming, occurring prior to the first cell division, is

141 a critical component of the high fidelity observed in this developmental decision.

142 In this study, we use VPC fate patterning to investigate the long-standing question of the

143 relationship between Ras and Rap1. We used CRISPR to fluorescently tag the endogenous RAP-

1441 and found it to be expressed throughout the animal and localized to plasma membranes and with

145 increased concentration at cell-cell junctions. We find that deletion of RAP-1 in an otherwise wild-

146 type background result in mild and low penetrance VPC patterning defects. Use of sensitized

147 genetic backgrounds indicated a cell-autonomous role for RAP-1 in promoting $1^{\circ}$ fate. CRISPR-

148 mediated mutational activation of endogenous RAP-1 revealed that RAP-1 is sufficient to induce 
149 ectopic $1^{\circ}$ cells, and also induce duplication of the excretory duct cell, another cell induction event 150 dependent on LET-60/Ras-LIN-45/Raf signaling. We found that the PXF-1/PDZ-GEF is also

151 required for maximal $1^{\circ}$ induction, consistent with a role for PXF-1 as the activating RAP-1 GEF

152 in VPC patterning. Furthermore, we find that during response to EGF signal, transgenic GFP

153 expression from the $p x f-1$ promoter changes from uniform expression across VPCs to increased in

154 presumptive $1^{\circ}$ cells while decreased in presumptive $2^{\circ}$ cells. This observation is another

155 confirmation of the re-programming hypothesis and suggests that the activation of RAP-1 is

156 restricted to $1^{\circ}$ cells during the phase when initial patterning of VPCs is reinforced. Taken together,

157 our results support a model in which a PXF-1-RAP-1 signal functions as a minor parallel input

158 into the major LET-60-LIN-45 $1^{\circ}$-promoting signaling.

Materials and Methods

161 C. elegans handling and genetics

162 All strains were derived from the parent N2 wild-type strain. Animals were grown at $20^{\circ} \mathrm{C}$ 163 under standard culturing conditions on NGM agar plates seeded with OP50 bacteria, unless stated 164 otherwise (Brenner 1974). Crosses were performed using standard methods. Strain details are 165 shown in Supplementary Table 1.

166 The let-60(n1046gf) strain undergoes genetic drift upon continuous growth, resulting in 167 increased phenotype strength (Zand et al. 2011). We therefore established firm guidelines to ensure 168 consistent results. Immediately upon thawing or construction, strains harboring $n 1046$ were scored 169 and then starved and parafilmed as a reference strain. For subsequent experiments, we 170 reestablished growing strains each week to avoid drift while in continuous culture. Experiments 171 were only reported if the let-60(n1046gf) control was within the well-validated baseline levels of 
172 induction. We did not observe any similar drift in rap-1(re180gf) or let-23(sa62gf) strains. Strains

173 harboring homozygous let-23(sa62gf) mutations did exhibit delayed growth, necessitating scoring

$174 \quad$ VPC induction a day later than with other strains.

175

Plasmids, Generation of CRISPR strains

177 Details of plasmid constructions are available upon request. Plasmids used are shown in 178 Supplementary Table 4. sgRNA sequences and repair templates are listed in Supplementary Tables

1795 and 6, respectively. The rap-1(re180gf) CRISPR strain was generated using the co-CRISPR 180 strategy (Paix et al. 2014) by microinjection of pJA58 (50 ng/ul), pNR21 (50 ng/ul), rap-1 (G12V)

181 ssODN repair template $(500 \mathrm{uM}), d p y-10($ cn64), ssODN repair template $(500 \mathrm{uM}))$ and co182 injection marker pPD118.33 (20 ng/ul) into N2 wild-type animals. Primers and conditions for PCR 183 genotyping are listed in Supplementary Table 3. PCR detection of rap-1(re180gf) was determined 184 using an overnight digestion with BamHI (NEB), NEB Cutsmart buffer, and water to a final 185 volume of $50 \mathrm{ul}$.

Scoring vulval induction and fate reporters (VPC and excretory duct)

188 To score the formation of normal and ectopic vulval induction, late L4 animals were mounted 189 in M9 on slides with a $3 \%$ NG agar pad containing $5 \mathrm{mM}$ sodium azide and examined by 190 DIC/Nomarski optics (Nikon eclipse Ni). To ensure accuracy and reproducibility, all presented 191 data are from animals grown together and are representative non-pooled samples. Presence of the $1921^{\circ} \mathrm{VPC}$ transcriptional reporters $\operatorname{arIs} 92\left[\mathrm{P}_{\text {egl-17 }}:\right.$ CFP-LacZ] and $\operatorname{arIs} 131\left[\mathrm{P}_{\text {lag- } 2: \because 2 x N L S: \because Y F P]}\right.$ were 193 scored for each of the six VPCs with the Nikon Eclipse Ni microscope, with a Nikon DS-Fi2 color 194 camera and using the NIS Elements Advanced Research, Version 4.40 (Nikon) software package. 
195 L3 animals were scored at the Pn.px (two-cell) stage to ensure that animals were past the point of 196 EGF induction of VPCs. Using the same equipment, newly hatched L1 larvae were scored for the 197 presence of a single or duplicated excretory duct cell(s) using the $\operatorname{sals} 14\left[\mathrm{P}_{l i n-48:: \mathrm{GFP}]}\right.$ transgene 198 (Johnson et al. 2001).

\section{RNA interference (RNAi)}

201 Bacterially mediated RNAi experiments were conducted at $23^{\circ} \mathrm{C}$ on NGM agar plates 202 supplemented with $1 \mathrm{mM}$ IPTG and $50 \mu \mathrm{g} / \mathrm{ml}$ carbenicillin as described (Kamath and Ahringer 203 2003). In our hands, we typically obtain more robust RNAi results at $23^{\circ} \mathrm{C}$ than at $20^{\circ} \mathrm{C}$ (Zand et 204 al. 2011). All RNAi clones were sequenced to confirm identity. RNAi plates were seeded with 80 $205 \mu$ HT115 bacteria harboring clones of $C$. elegans genes (Source Bioscience) or the negative control luciferase (luc; Shin et al., in preparation) and allowed to grow overnight at room 207 temperature. Late L4 larvae were added to each plate and transferred to a fresh RNAi plate the 208 next day. Founding parents were then removed the following day. Late L4 progeny were scored 209 for the formation of the principal vulva and/or ectopic pseudovulvae by DIC/Normarski optics 2 210 days later. However, as noted above, strains homozygous for the let-23(sa62gf) allele reach

211 maturity at a slower rate, and consequently were scored 4 days after parents were removed. For 212 each experiment, pop-1(RNAi), which confers $100 \%$ embryonic or L1 lethality under optimal 213 conditions, was used as a control for maximal RNAi efficacy; animals were only scored from 214 experiments in which $100 \%$ lethality was observed. RNAi strains used are included in 215 Supplemental Table 2.

216 VPC-specific RNAi was performed using the genotype let-23(sa62gf); rde-1(ne219); 217 mfIs70[Plin-31::rde-1(+), $P_{m y o-2:: g f p] .}$ The mfIs70[Plin-31::rde-1(+), $P_{m y o-2:: g f p] ~ i n t e g r a t e d}$ 
218 transgene which rescues the loss of $r d e-1$ specifically in the VPCs (Barkoulas et al. 2013) was

219 introduced and tracked in crosses using the dpy-11(e224) unc-76(e911) chromosome in trans as a

220 balancer.

221

222

Fluorescent microscopy (imaging)

Animals were mounted live in M9 buffer on slides with a 3\% agar pad containing $5 \mathrm{mM}$ sodium

azide. DIC/Nomarski optics and epifluorescence microscopy were captured using the Nikon

225 Eclipse Ni and confocal images using the A1si Confocal Laser Microscope (Nikon). Captured

226 images were processed using NIS Elements Advanced Research, Version 4.40 (Nikon) and Adobe

227 Photoshop CC 2018 software packages (inverted images).

228

229 Plasmids and strains are available upon request. The authors affirm that all data necessary for

230 confirming the conclusions of the article are present within the article, figures, and tables.

\section{Results}

\section{RAP-1 is expressed in VPCs and localized to plasma membrane and junctions}

234 The Ras superfamily of small GTPases is characterized by lipid modification of their C-

235 terminal CAAX motif, resulting in their subcellular localization to the lipid bilayers. For Ras itself,

236 this primarily means localization to the plasma membrane (Hancock et al. 1990; Prior and Hancock

237 2012; Reiner and Lundquist 2016). Likewise, Rap1 has been observed to be found on the plasma

238 membrane (Pizon et al. 1994). Prior work reported LET-60/Ras to ubiquitously expressed in $C$.

239 elegans (Dent and Han 1998). Although the let-60/Ras promoter fusion could not provide

240 information regarding subcellular localization, ectopic induction of $1^{\circ} \mathrm{s}$ conferred by the let- 
60(n1046gf) activating mutation was reversed by treatment of farnesyl transferase inhibitors, which block C-terminal prenylation and hence activity (Hara and Han 1995). Consequently, we are reasonably confident that $C$. elegans LET-60 is targeted to the plasma membrane.

244 To determine the expression pattern and subcellular localization of endogenous RAP-1, we used CRISPR-Cas9-mediated genome editing to insert an mNeonGreen::3xFlag epitope tag into the 5 ' end of the endogenous rap-1 gene, generating rap-1(cp151[mNeonGreen^3xFlag::rap-1])

247 (Fig. S3; Dickinson et al. 2015). In keeping with previous observations with let-60, tagged RAP2481 expression was ubiquitous throughout the animal during development, and localized to the 249 plasma membrane (Fig. S4). Of particular importance to this study, RAP-1 expression was 250 observed in the VPCs prior to EGF induction (1 cell stage, Pn.p; Fig. 2A,C), after EGF induction 251 (2 cell stage, Pn.px; Fig. 2B,D), and throughout vulval morphogenesis.

252 We also observed that tagged endogenous RAP-1 is localized to cell-cell junctions between 253 hypodermal seam cells (Fig. S4A). This observation is consistent with the established role of RAP2541 in the assembly of cadherin complexes at junctions during embryogenesis, a process that is 255 redundant with another small GTPase, RAL-1 (Frische et al. 2007), and our observation that tagged 256 endogenous RAL-1 also localizes to the plasma membrane and cell-cell junctions (Shin et al., 257 submitted). This finding also conforms with the established role of Rap1 in junctional biology in 258 Drosophila and mammalian cell culture (Caron et al. 2000; Reedquist et al. 2000; Knox and Brown 259 2002).

260 Intriguingly, we observed increased expression of tagged endogenous RAP-1 in the anchor cell 261 (AC). The $\mathrm{AC}$ is known to undergo polarized invasive behavior, directed toward the P6.p $/ 1^{\circ}$ 262 lineage of the vulva (Sherwood and Sternberg 2003; Hagedorn et al. 2009; Ziel et al. 2009) Fig. 263 2C,D). We additionally observed increased expression of RAP-1 in the distal tip cell (DTC) of the 
264 developing somatic gonad (Fig. S4B), a tissue that also goes through invasive migration. However,

265 our later experiments indicate that RAP-1 functions cell autonomously to regulate VPC fate

266 patterning, so we interpret the increased RAP-1 expression in the AC to be unrelated to VPC fate

267 patterning.

268

269 RAP-1 is necessary for maximal induction of $1^{\circ}$ VPCs

270 The early false lead in mammalian cell culture of Rap1 as a competitive inhibitor of Ras has

271 long muddied the waters of our understanding of the role of Rap1 in Ras signaling (Kitayama et

272 al. 1989; Kitayama et al. 1990; Sakoda et al. 1992; Cook et al. 1993; see Introduction).

273 Additionally, investigations of Rap1 have been complicated by its multiple isoforms in vertebrates

274 and its essential role in development in Drosophila (Mishra et al. 2005). Previous work showed $C$.

275 elegans rap-1 putative null mutant animals to be fertile and viable, including

276 mNeonGreen ${ }^{\wedge}$ SEC $3 x$ Flag::rap-1 animals retaining the SEC positive/negative selection cassette,

277 which should disrupt RAP-1 expression (Frische et al. 2007; Dickinson et al. 2015). We more

278 closely examined animals mutant for two independent rap-1 strong loss or null alleles. Both rap-

2791 (tm861) (a deletion that results in a frame shift) and rap-1(pk2082) (nonsense allele in exon 5 of

280 6; Fig. S3) conferred low penetrance abnormal vulval patterning, which we interpret to be missing

$28122^{\circ}$ cells (Fig. S5). This phenotype is similar to that observed in animals mutant for the hypomorphic

282 lin-3(e1417), let-60(n2021) and lin-45(n2506) mutations, with reduction of function of EGF, Ras

283 and Raf orthologs, respectively (Wang and Sternberg 1999; Zand et al. 2011). Retrospectively, we

284 interpret this low penetrance under-induction of $2^{\circ}$ cells to be due to weak specification of $1^{\circ}$ cells.

285 Indeed, the mutations mentioned here, which confer phenotypes stronger than those of rap-1

286 mutations, frequently confer a lack of $1^{\circ}$ cells altogether. We speculate that these under-induced 
$1^{\circ}$ cells express insufficient levels of the triply redundant DSL ligands, LAG-2, APX-1, and DSL-

1 (Chen and Greenwald 2004) Zhang and Greenwald 2011) to reliably induce the neighboring

VPCs to become $2^{\circ}$.

The low penetrance of these rap-1 mutant defects makes the proper interpretation of the

291 function of RAP-1 in VPC fate patterning difficult. Hence, we used a sensitized genetic

292 background, let-23(sa62gf) (Katz et al. 1996), which is sufficient to induce ectopic $1^{\circ}$ s and are

294 resulted in significantly decreased ectopic $1^{\circ}$ s compared to let-23(sa62gf) alone (Fig. 3A). We also

295 targeted rap-1 loss in a VPC-specific manner using the let-23(sa62gf); rde-1(ne219) background

296 with the mfIs70 integrated transgene (Plin-31 driving VPC-specific rescue with $r d e-1(+))$. In

297 agreement with our previous findings, VPC-specific depletion of rap-1 resulted in a significant

298 decrease in the formation of ectopic $1^{\circ} \mathrm{s}$ (Fig. 3B). These results demonstrated that RAP-1

299 functions cell-autonomously in the VPCs. To further evaluate the effects of rap-1 loss, we utilized

300 the let-60(n1046gf) mutant strain, which harbors the G13E mutation analogous to mutation in

301 mammalian Ras that lock the protein in the GTP-bound form, thus causing the protein to be

302 constitutively active (Han et al. 1990; Beitel et al. 1990). Similar to our findings with let-

303 23(sa62gf), above, rap-1(tm861) reduced ectopic $1^{\circ}$ induction in the let-60(n1046gf) background

304 (Fig. 3C). Consequently, we hypothesize that RAP-1 functions in parallel to or downstream of

305 LET-60 to promote $1^{\circ}$ fate. Given the biochemical relationships of mammalian Rap1 and Ras to

306 Raf, we favor an interpretation of parallelism, potentially converging on LIN-45/Raf.

307 We previously noted that let-60(n1046gf), which is predicted to be GAP insensitive, was

308 paradoxically still responsive to RNAi-mediated depletion of the negative regulator, GAP-1 (Zand

309 et al. 2011). We reproduced this result, observing that the let-60(n1046gf) single mutant responded 
310 to gap-1(RNAi) by increasing ectopic $1^{\circ}$ induction, with RNAi targeting the LIP-1/ERK

311 phosphatase (Zand et al. 2011) as a positive control and luciferase-directed RNAi as a negative

312 control (Fig. 3D). However, we found that rap-1(tm861) let-60(n1046gf) double mutant animals

313 no longer responded to gap-1(RNAi) while still responding to lip-1(RNAi) (Fig. 3D). These results

314 suggest that GAP-1 functions to promote GTP hydrolysis of both RAP-1 and LET-60/Ras related

315 small GTPases. Accordingly, the mammalian GAP1 subfamily of GAPs, orthologous to C. elegans

316 GAP-1 (Stetak et al. 2008; Grewal et al. 2011), has been shown to be bifunctional, targeting

317 mammalian Ras as well as Rap1 and Rap2 as substrates (Kupzig et al. 2006).

\section{RAP-1 is sufficient to induce ectopic $1^{\circ}$ cells}

If RAP-1 functions in parallel to LET-60 to activate LIN-45/Raf and hence induction of $1^{\circ}$

321 cells, we would expect constitutively active RAP-1 to promote induction of ectopic $1^{\circ}$ cells. We

322 used CRISPR/Cas9 genome editing to introduce the classical G12V activating mutation into the

323 endogenous rap-1 locus (Fig. 4A). The G1 region in both Ras/Rap1 proteins is exceptionally well

324 conserved across species (Fig. S1,2; Wennerberg et al. 2005; Reiner and Lundquist 2016) allowing

325 the G12V mutation to be successfully used for both Ras and Rap1 in C. elegans (Pellis-van Berkel

326 et al. 2005; Zand et al. 2011) and Drosophila (Mishra et al. 2005), as well as mammalian cell

327 culture (Vossler et al. 1997). The rap-1(re180gf) allele can be detected by PCR and restriction

328 enzyme digest due to the introduction of the concomitant silent BamHI site (Fig. 4B).

rap-1(rel80gf) is sufficient to promote ectopic $1^{\circ}$ cell differentiation (Fig. 4C, D, S6A). As

330 predicted based on let-60(n1046gf) (Beitel et al. 1990; Han et al. 1990), the rap-1(re180gf)

331 mutation acts in a gain-of-function manner, evidenced by semi-dominant induction of ectopic $1^{\circ}$

332 cells (Fig. 4E). Depletion of the LIP-1/ERK phosphatase can increase ectopic $1^{\circ}$ induction in 
sensitized backgrounds (Berset et al. 2001; Yoo et al. 2004; Berset et al. 2005 Zand et al. 2011),

failed to enhance the ectopic $1^{\circ}$ induction in the rap-1(rel80gf) background (Fig. 4F).

To verify that rap-1(re180gf) was promoting $1^{\circ} \mathrm{VPC}$ transcriptional programs, we utilized two

17::CFP-LacZ, Pttx-3::GFP) (Yoo et al. 2004; Zhang and Greenwald 2011). In control animals,

we also observed YFP in both P5.px and P7.px (Fig. 5A-D). A similar increase in ectopic

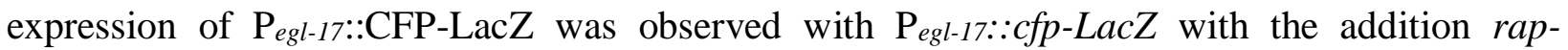

342 1(re180gf) (Fig. S6B-E). For both expression of $\mathrm{P}_{\text {lag- } 2:: Y F P}$ and $\mathrm{P}_{\text {egl-17::CFP-LacZ the addition of }}$

343 rap-1(rel80gf) resulted in a significant increase expression outside of P6.px. Rare expression in

344 and transformation of $3^{\circ}$ VPCs was also observed (data not shown; Fig. S6A).

\section{RAP-1 is sufficient to promote excretory duct cell duplication}

We observed that some rap-1(rel80gf) animals exhibited a ventral protrusion near the 348 posterior bulb of the pharynx (Fig. 6B). let-60(n1046gf) confers a similar ventral protrusion, which

352 the excretory duct cell to develop, and hence the inference of inability to excrete solutes (Beitel et 353 al. 1990; Han and Sternberg 1990; Han et al. 1990; Yochem et al. 1997). To determine if RAP-1

354 activation is sufficient to result in excretory duct cell duplication, we used the saIs $14\left(\mathrm{P}_{\text {lin-48::GFP) }}\right.$

355 transgene, a cell fate reporter for the duct cell (Johnson et al. 2001). Mirroring our results in VPC 
cell fate patterning, rap-1(rel80gf) was sufficient to drive duct-cell duplication, but at a reduced rate in comparison to let-60(n1046gf) (Fig. 6). We observed no rod-like larval lethality in rap1(tm861) or rap-1(pk2082) mutants, suggesting that rap-1 is not necessary for induction of the

359 excretory duct cell.

\section{PXF-1/RapGEF is required for maximal induction of $1^{\circ}$ cells}

The Ras superfamily of small GTPases are tightly regulated by GEFs, which promote GTP loading and hence active conformation, and by GAPs, which promote GTP hydrolysis to GDP and hence inactive conformation (Wennerberg et al. 2005). While certain mammalian GAP and GEF families have overlapping specificity for both Ras and Rap1, each also maintains unique regulators. Our results (Fig. 3D, above) suggest that GAP-1/GAP1 functions to repress RAP-1 as well as the published LET-60/Ras (Hajnal et al. 1997; Stetak et al. 2008). essential for development. Loss of $p x f-1$ function results in hypodermal defects, inability to molt,

371 and variable onset larval lethality. Consistent with redundant roles for RAP-1 and RAP-2 in

372 molting, loss of either individually results in live, superficially wild-type animals. However, loss 373 of both rap-1 and rap-2 phenocopies loss of pxf-1, suggesting that PXF-1 functions as a joint GEF

374 for both RAP-1 and RAP-2, as is seen in mammals (Pellis-van Berkel et al. 2005; Raaijmakers and 375 Bos 2009).

376 To determine potential activators of RAP-1 in VPC patterning we assayed putative RapGEFs, $377 p x f-1$ and Y34B4A.4, that are expressed in tissues other than neurons (Wormbase release WS262).

378 Due to the essential role of PXF-1 in development, we again utilized VPC-specific RNAi in a 


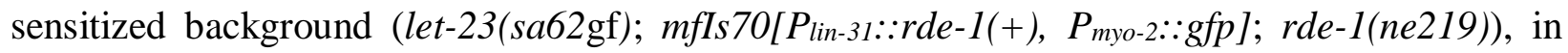
which the RNAi defective phenotype of the $r d e-1$ mutation is rescued by VPC-specific expression

381 of wild-type RDE-1 (Barkoulas et al. 2013). VPC-specific pxf-1(RNAi) (Fig. 7F) but not 382 Y34B4A.4(RNAi) (Fig. S7A) resulted in decreased ectopic $1^{\circ}$ induction. These findings suggest 383 that the PXF-1 RapGEF promotes $1^{\circ}$ fate, consistent with it functioning as a GEF that activates 384 RAP-1 in VPCs.

\section{Expression from the $p x f-1$ promoter is dynamically restricted to $1^{\circ}$ cells.}

While both RAP-1 and LET-60 are sufficient to induce transformation of ectopic $1^{\circ}$ cells, our results suggest such they differ both in strength and spatial activity. Unlike constitutively activated LET-60/Ras, which transforms VPCs that normally become $3^{\circ}$ into $1^{\circ}$ cells, constitutively activated RAP-1 mostly transforms presumptive $2^{\circ}$ cells (P5.p and P7.p) to become $1^{\circ}$ (Fig. 5D,

Fig S6E). This observation suggested that the activity of RAP-1 is more spatially restricted within in the VPCs than is the activity LET-60, consistent with the modulatory role of RAP-1 compared to the central role of LET-60. Yet, like LET-60 (Dent and Han 1998), RAP-1 expression is uniform

394 throughout VPCs (Fig. 2A-D). Consequently, we examined the expression of the putative GEF for RAP-1, PXF-1.

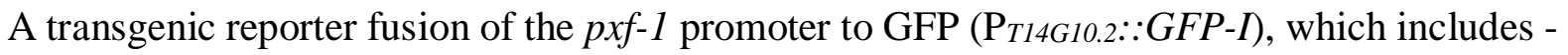
3972394 to +26 relative to the translational start codon of $p x f-1$ exon 1 , expressed GFP in the 398 hypodermis (Pellis-van Berkel et al. 2005). We evaluated dynamic $\mathrm{P}_{T 14 G 10.2:: G F P-I}$ expression in 399 VPCs before and after onset of LIN-3/EGF induction using the first cell division (Pn.p to Pn.px) 400 as a conservative temporal indicator of induction. Prior to LIN-3/EGF induction, we observed 401 uniform GFP expression in all VPCs (Fig. 7B, C). After induction, we observed GFP expression 
402 to be increased in P6.p, the presumptive $1^{\circ}$ cell, and absent in P5.p and P7.p, the presumptive $2^{\circ}$

403 cells (Fig. 7D, E). We therefore hypothesize that $p x f-1$ expression is dynamically altered in

404 response to the onset of LIN-3/EGF expression. We propose that this regulatory mechanism serves

405 to restrict RAP-1 activation to the presumptive $1^{\circ}$ cell, while abrogating RAP-1 activation in 406 presumptive $2^{\circ}$ cells (Fig. 1).

407

408 Discussion

409 Our genetic results suggest that RAP-1 functions cell autonomously to promote $1^{\circ}$ fate. Our 410 results also suggest that RAP-1, in addition to LET-60, is a substrate of the bifunctional GAP-

411 1/GAP1. Critically, the constitutively activated endogenous RAP-1 mutant is semi-dominant and

412 is sufficient to induce $1^{\circ}$ fate. And, like let-60(gf), rap-l(gf) duplicates excretory duct cells. Yet

413 the ectopic $1^{\circ}$ induction and excretory duct cell duplication phenotypes conferred by rap- 1 (gf) are

414 substantially weaker than those of let-60(gf). These observations indicate that, as expected, LET-

41560 plays a central role while RAP-1 plays a modulatory role. (It is worth noting that the G12V

416 mutation that we use to activate RAP-1 was never isolated for LET-60 in screens, presumably

417 because full LET-60 activation is predicted to confer both lethality (Schutzman et al. 2001; Pellis-

418 van Berkel et al. 2005) and sterility (Eisenmann and Kim 1997). G13 mutations are predicted to

419 be weaker in their activation of GTPases (Reiner and Lundquist 2016).

420 We also found that PXF-1/PDZ-GEF, a Rap1GEF in mammalian cells and in C. elegans, is 421 required to maximally promote $1^{\circ}$ fate. This function, like that of RAP-1, is also cell autonomous 422 and suggests that PXF-1 functions as the GEF that triggers RAP-1 activation in VPCs. Activation 423 upstream of PXF-1 remains unknown. Interestingly, like many other signaling genes in the VPC 424 signaling network (see Introduction), expression from a $p x f-1$ promoter::GFP reporter changes 
425 after initial induction. Expression prior to EGF induction is uniform throughout the VPCs, but after 426 induction GFP signal increases in the presumptive $1^{\circ}$ cell and is extinguished from presumptive $2^{\circ}$

427 cells. Accordingly, we hypothesize that as a consequence of transcriptional reprogramming of $p x f$ -

4281 expression, the potential for RAP-1 activation is increased in presumptive $1^{\circ}$ cells and decreased 429 in presumptive $2^{\circ}$ cells (Fig. 1 ).

430 RAP-1 may be activated by EGF in the same cells as LET-60, i.e. those VPCs closest to the 431 EGF source, the AC. Yet we propose that the functions of LET-60 and RAP-1 are qualitatively 432 different. The quantitatively weaker rap-1(re180gf) confers mostly $2^{\circ}$-to- $1^{\circ}$ transformations (this 433 study), while the stronger let-60(n1046gf) confers mostly $3^{\circ}$-to- $1^{\circ}$ transformations: $2^{\circ}$ s are rarely 434 transformed or express markers of ERK activation in the let-60(n1046gf) background (Beitel et al. 435 1990; Han et al. 1990; Berset et al. 2001; Yoo et al. 2004; Zand et al. 2011). For let-60(n1046gf) 436 to transform $2^{\circ}$ cells to $1^{\circ}$ cells, it takes dramatic derepression of the EGFR-Ras-Raf MEK-ERK $4371^{\circ}$-promoting cascade, by simultaneous inactivation of $2^{\circ}$-specific LIP-1/ERK phosphatase and 438 DEP-1/EGFR phosphatase (Berset et al. 2005) or reduction of $2^{\circ}$-promoting signals (Yoo et al. 439 2004; Yoo and Greenwald 2005; Zand et al. 2011). These differences between LET-60 and RAP4401 beyond strength of transformation may reflect different regulatory mechanisms between the two.

441 For example, LET-60 is thought to be activated by the RasGEF SOS-1 (Chang et al. 2000; 442 Modzelewska et al. 2007), while our data suggest that RAP-1 is activated by PXF-1. Conversely, 443 while GAP-1 and GAP-3/RASA1 redundantly inhibit $1^{\circ}$ fate induction, mammalian isoforms of 444 both are potentially bifunctional (Kupzig et al. 2006; Raaijmakers and Bos 2009), and their relative 445 contributions to LET-60 and RAP-1 inactivation are unclear.

446 RAP-1 mutant phenotypes and re-programming of expression from the $p x f-1$ promoter after 447 EGF induction are consistent with other transcriptional re-programming events in vulva signaling. 
448 We hypothesize that many levels of such regulation impose higher developmental fidelity on the 449 system: a collective re-configuration of signaling protein expression could refine the action of 450 signals, thus providing the transition from a ligand gradient to discrete binary outputs. In other 451 words, re-configuration of signals could decrease the potential for developmental noise inherent 452 in a gradient, or contradictory signals in the same cell.

453 Our results are also consistent with the role of Rap1 in developmental signaling as a "shadow 454 Ras" that echoes and reinforces the main function of Ras signaling through Raf. It is difficult, 455 however, to prove that RAP-1 signals through LIN-45/Raf. Yet our results are consistent with this 456 model, as are the molecular characteristics of RAP-1 as a close relative of LET-60. However, an 457 alternative model is that RAP-1 acts to reinforce other aspects of the $1^{\circ}$-promoting signal. For 458 example, RAP-1 could promote or stabilize EGFR signaling, as Drosophila Rap1 has been shown 459 to regulate EGFR membrane localization and polarity through its junctional effector 460 Canoe/afadin/AF6 (O'Keefe et al. 2009; Baril et al. 2014). Such a function, piggybacking on the 461 strong induction of $1^{\circ}$ cells by LET-60, could induce occasional ectopic $1^{\circ}$ cells.

462 Our study provides mechanistic insights into the function of RAP-1, the C. elegans ortholog 463 of mammalian Rap1 proteins that function as putative human oncogenes. We additionally establish 464 a role for the RapGEF PXF-1 as the putative upstream GEF for RAP-1 in VPC fate patterning, and 465 argue that transcriptional control of the $p x f-1$ promoter over time provides an example of how the 466 VPC signaling network is dynamically re-programmed during development to prevent potentially 467 conflicting signals. We hypothesize that PXF-1-RAP-1 functions as a modulatory parallel input to 468 reinforce and spatially restrict LET-60-LIN-45-dependent $1^{\circ}$ cell induction. 


\section{Acknowledgments}

473 This work was supported by NIH grants R01-GM121625 and R21-HD090707 to D.J.R, ACS PF-

474 16-083-01 post-doctoral fellowship to N.R and a Howard Hughes Postdoctoral Fellowship from

475 the Helen Hay Whitney Foundation to D.J.D. Some strains were provided by the CGC, which is

476 funded by NIH Office of Research Infrastructure Programs (P40 OD010440). Wormbase was used

477 regularly. We thank the Reiner and Arur labs for helpful discussions. 
A

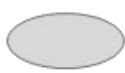

P3.p

B

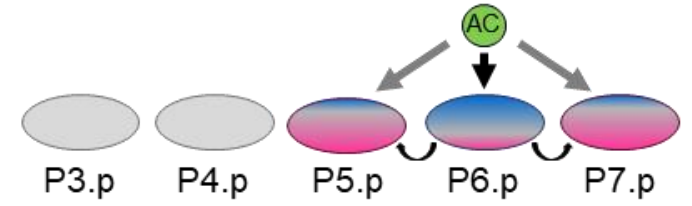

C

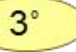

P3.p

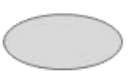

P4.p

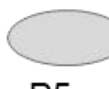

P6.p

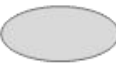

P7.p

P7.p

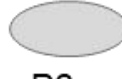

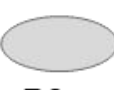
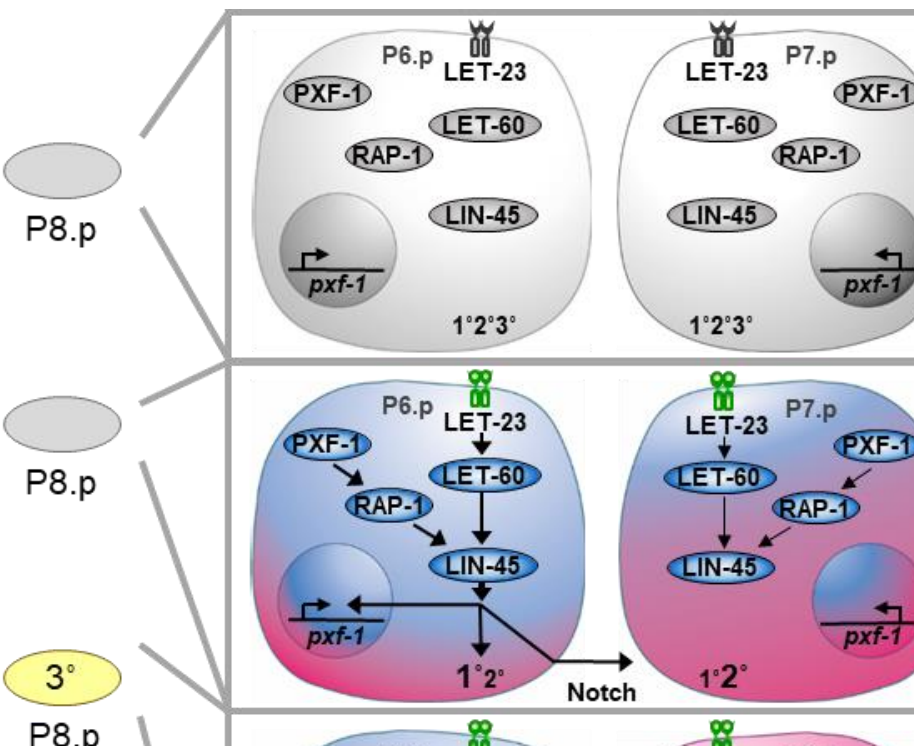

LIN-45

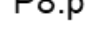

D $\begin{array}{ll}\text { LET-60 } & \text { MTEYKLVVVGDGGVGKSALTIQLIONHFVE PDPTIEDSYRKOVVIDGETCLLDILDTAG } \\ \text { RAP-1 } & \text { MREYRIVVLGSGGVGKSALTVQFVQGIFVEK YDPTIEDSYRKQVEVDGQOCMLEILDTAG }\end{array}$

$\begin{array}{ll}\text { LET-60 } & \text { QEEYSAMRDQYMRTGEGFLLVEAVNEAKSFENVANYREQIRRVKDSDDVPMVLVGNKCDL } \\ \text { RAP-1 } & \text { TEOFTAMRDLYMRNGQGFVLVYSITAQSTFNDLMDLRDQILRVKDTDEVPMILVGNKCDL }\end{array}$

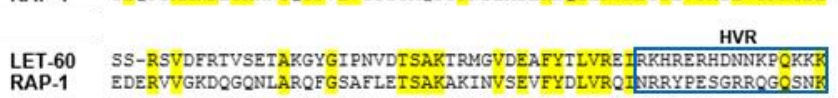

\begin{tabular}{lr}
$r$ CAAX \\
LET-60 \\
RAP-1 \\
\hline OCCSCVIM
\end{tabular}

Figure 1. A model of RAP-1 and vulval cell fate patterning

A) Patterning of the vulva begins with six equipotent vulval precursor cells (VPCs). B) Initial cell fate specification of the VPCs begins in response to the release of EGF from the nearby anchor cell (AC) through induction of the classic LET-60/Ras-LIN-45/Raf cascade and a subsequent lateral inhibitory signal via Notch. C) $1^{\circ}$ cell fate is then reinforced through the restricted and amplified expression of PXF-1, resulting in elevated RAP-1 activation. D) Sequence alignment of LET-60 and RAP-1 with the core effector binding sequence shaded in red and identical residues in yellow. Core effector binding sequences are thought to confer specificity for suites of effectors, and are generally identical across metazoans. The C-terminal hyper-variable and CAAX are outlined in the blue. 

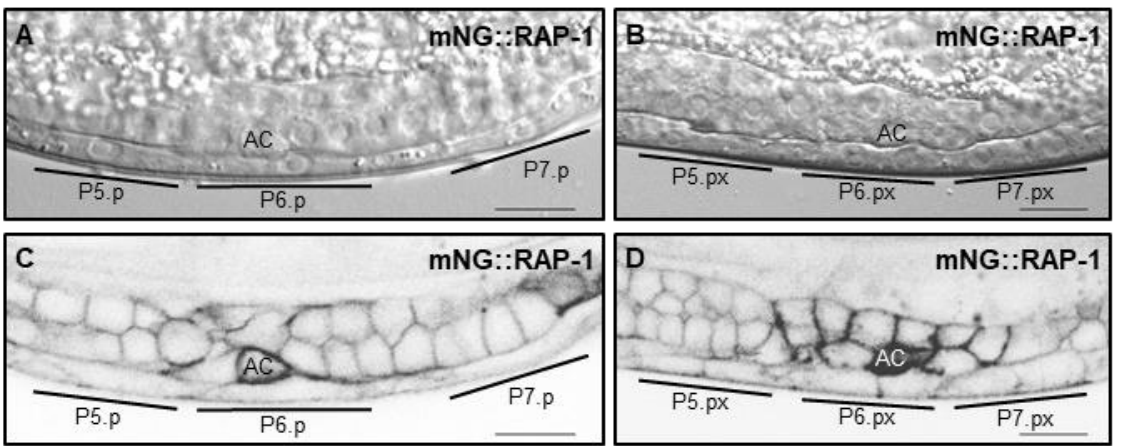

481 Figure 2. Endogenous RAP-1 is expressed throughout the VPCs

Representative DIC (A, B) and confocal fluorescence (C, D) micrographs of rap-1(cp151[mNG^3xFlag:::rap-1]) at $(\mathbf{A}, \mathbf{C})$ the 1-cell (Pn.p) and (B, D) 2-cell (Pn.px) stages."mNG" = mNeonGreen (Shaner, Lambert et al. 2013). Scale bar $=10 \mu \mathrm{m}$. 
A

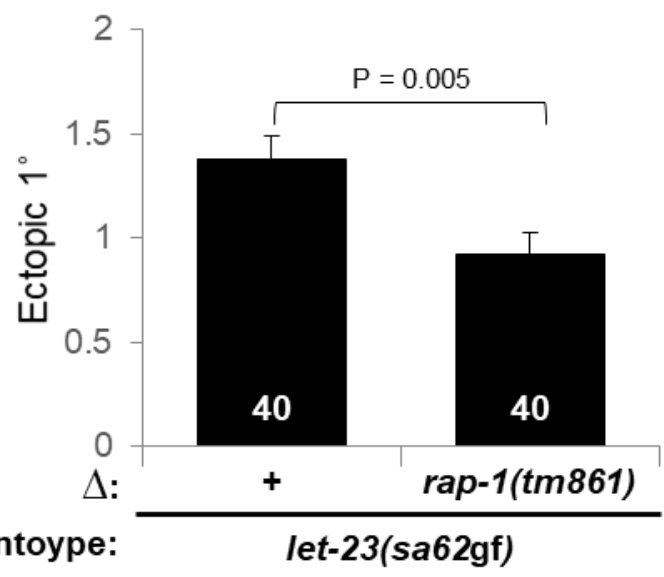

C

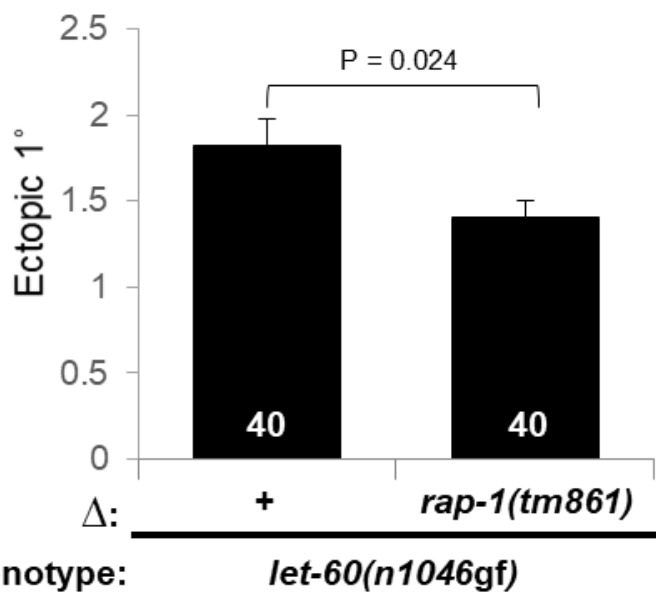

B

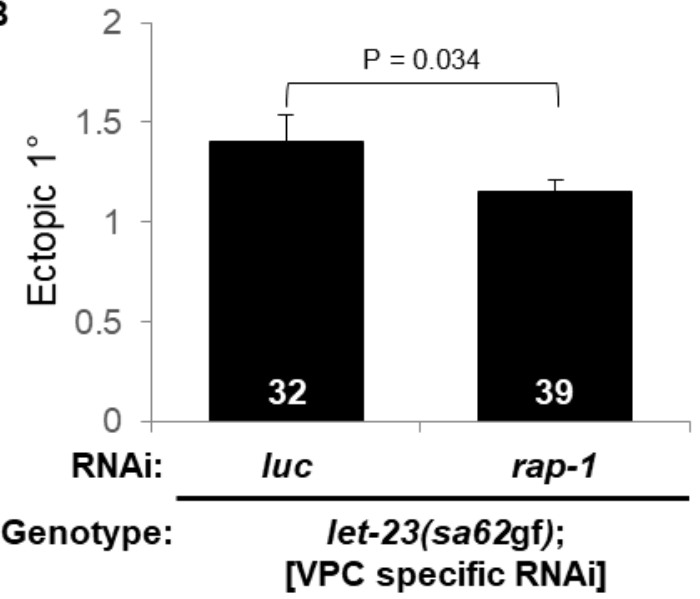

D

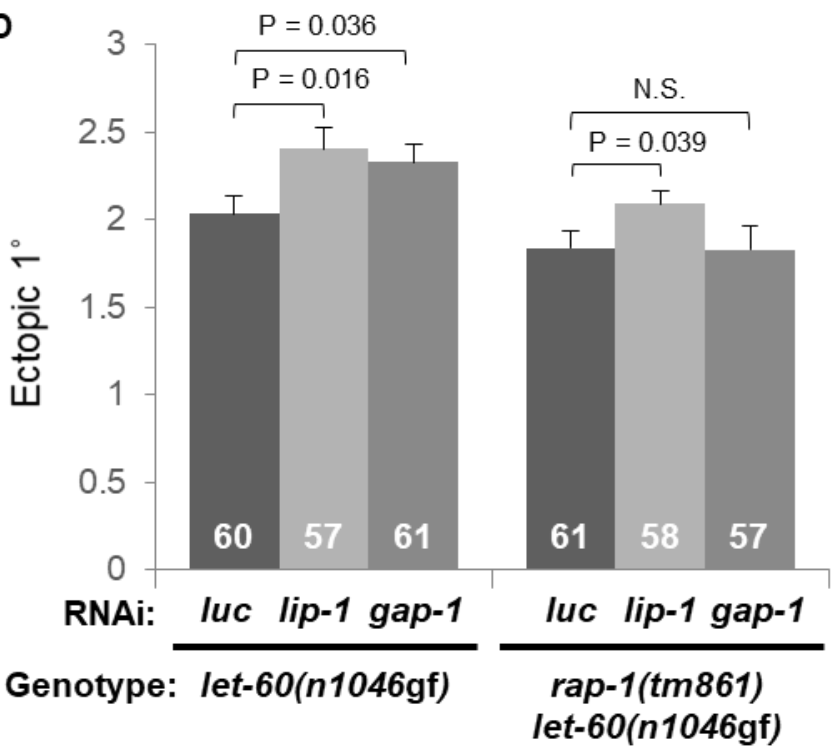

Figure 3. RAP-1 is necessary for maximal $1^{\circ}$ induction and confers response to GAP-1/RasGAP.

A) Deletion or B) VPC-specific RNAi knockdown of rap-1 decreased ectopic $1^{\circ}$ induction in the let-23(sa62gf) (cis-marked with unc-4(e120)) background. VPC-specific RNAi = rde-1(ne219); mfls70[ $\mathrm{P}_{\text {lin-31: }}:$ rde-1(+), $\mathrm{P}_{\text {myo- }}$ 2::gfp]. Luciferase-directed (Iuc) RNAi served as a negative control (Shin et al., submitted). C) rap-1 deletion decreased ectopic $1^{\circ}$ induction in the let-60(n1046gf) background. D) let-60(n1046gf) animals are sensitive to gap-1- and lip-1-directed RNAi (depletion of RasGAP and ERK phosphatase, respectively), while rap-1(tm861) let-60(n1046gf) animals are insensitive to gap-1(RNAi) but still responsive to lip-1(RNAi). All data are representative non-pooled assays, collected on the same day, of the mean ectopic $1^{\circ}$ cells \pm S.E.M. D) let60(n1046gf) vs. rap-1(tm861) let-60(n1046gf) animals were scored on separate days. Y-axes indicate number of ectopic $1^{\circ}$ cells. $\mathrm{N}=$ white numbers. $\mathrm{P}$ values were calculated by T-test or ANOVA. 
A

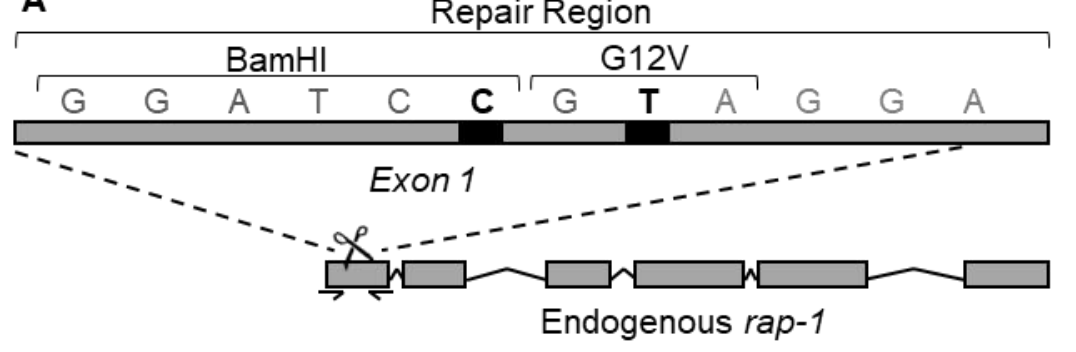

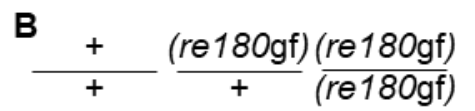
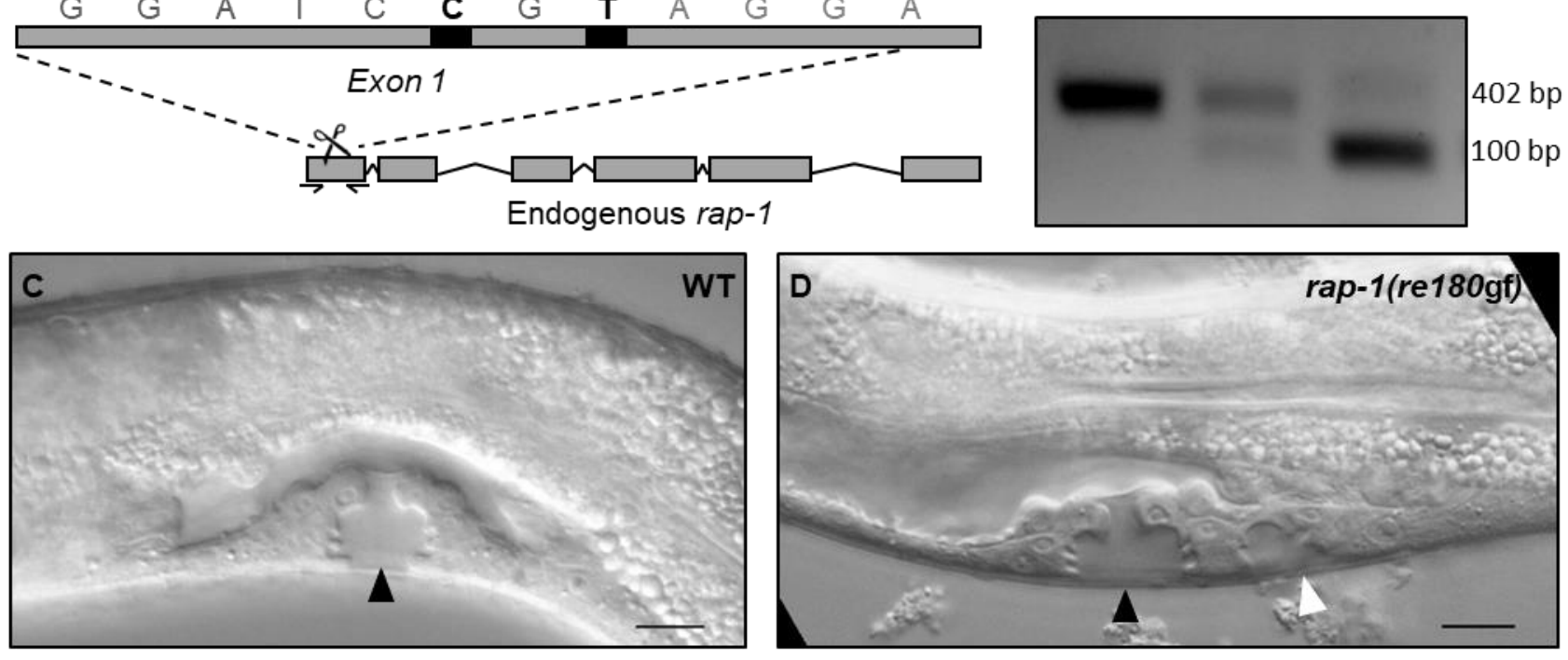

E

음<smiles>O=S(=O)(O)OC1CCCCC1</smiles>

Genotype:
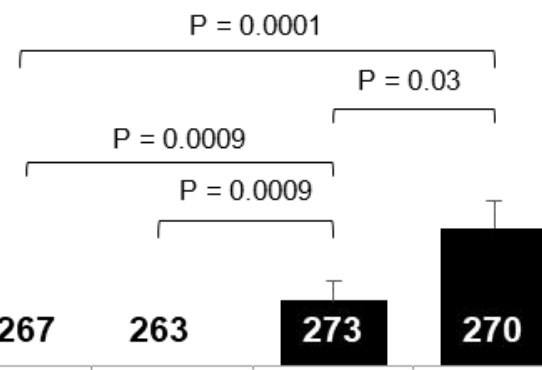

484

Figure 4. RAP-1 is sufficient to promote ectopic $1^{\circ}$ cells

A) Diagram of the strategy for CRISPR/Cas9-dependent knock-in of the activating (G12V) mutation into endogenous rap-1, along with a silent BamHI restriction site for genotyping. B) PCR genotyping of the rap1(re180gf) mutant allele, followed by BamHI digestion and agarose gel electrophoresis. C,D) Representative DIC micrographs at the late L4 stage shows the wild-type vulva (black triangle) in (C) wild-type (WT) animals, and induction of ectopic $1^{\circ} \mathrm{S}$ (white triangle) in (D) rap-1(re180gf). Scale bar $=10 \mu \mathrm{m}$. E) Both heterozygous and homozygous rap-1(re180gf) animals exhibited increased ectopic $1^{\circ}$ induction compared to WT. bal $=$ rap-1

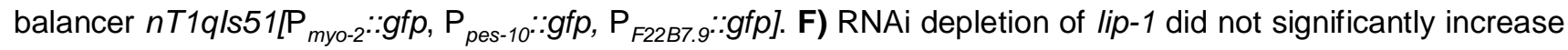
ectopic $1^{\circ} \mathrm{S}\left(\right.$ luc $=$ luciferase RNAi negative control). $\mathrm{Y}$ axis $=$ mean number of ectopic $1^{\circ} \mathrm{VPCs} \pm \mathrm{S} . \mathrm{E} . \mathrm{M} . \mathrm{N}=$ numbers on columns. $\mathrm{P}$ values were calculated by T-test. 


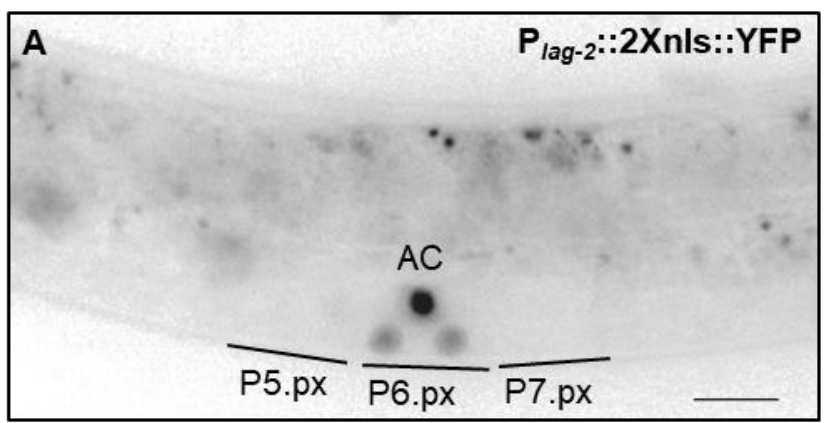

C

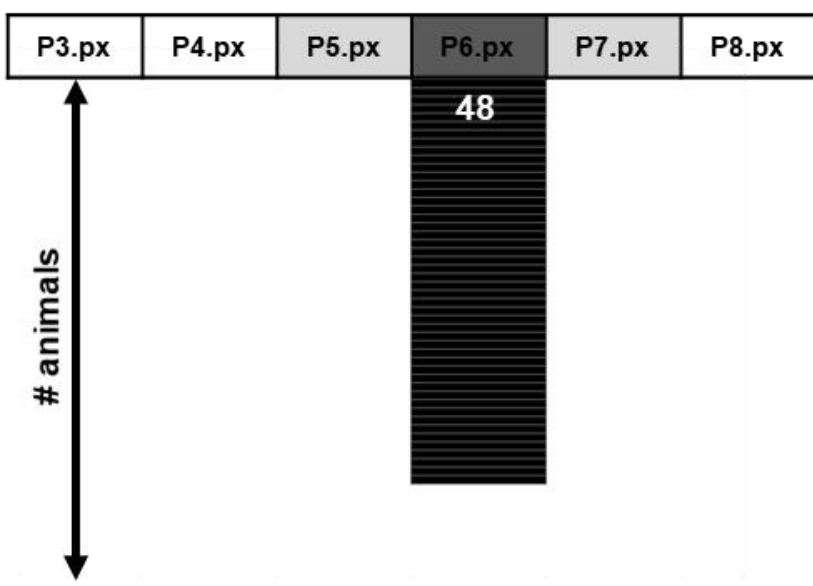

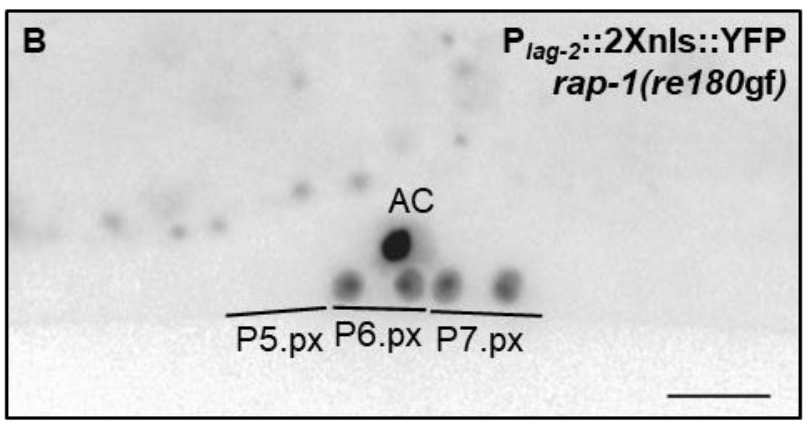

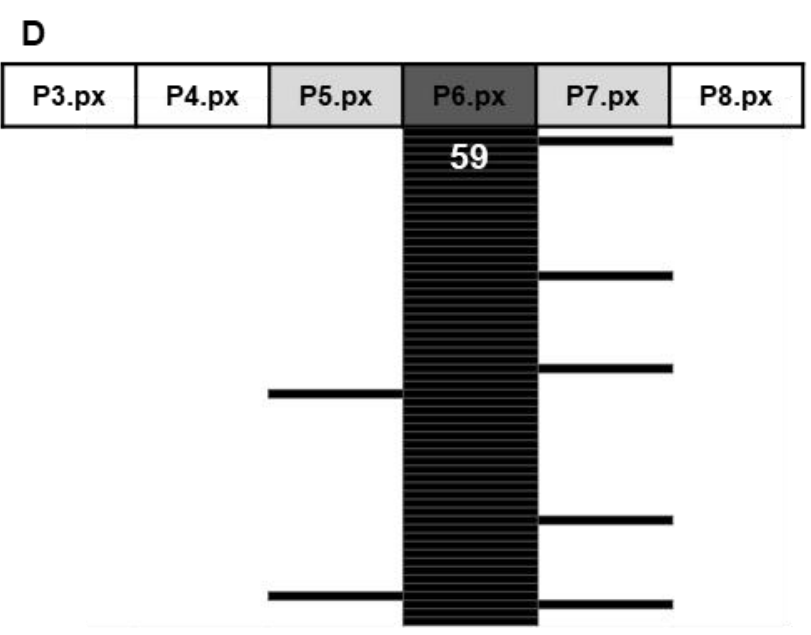

Figure 5. RAP-1 activation results ectopic expression of $1^{\circ}$ VPC transcriptional reporters

$\mathbf{A}, \mathbf{B})$ Representative epifluorescence micrographs in animals at the 2-cell (Pn.px) stage show the inappropriate

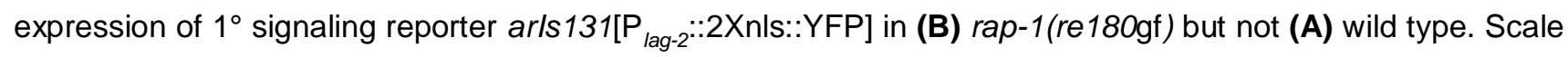

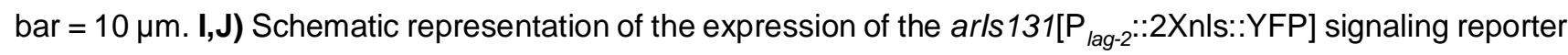
across the all six VPCs for both (C) control and (D) rap-1(re180gf). The addition of rap-1(re180gf) resulted in a significant increase of expression outside of P6.px $(P=0.016) P$ values were calculated by Fisher's Exact test. $N$ $=$ white number, with each line representing an animal. 

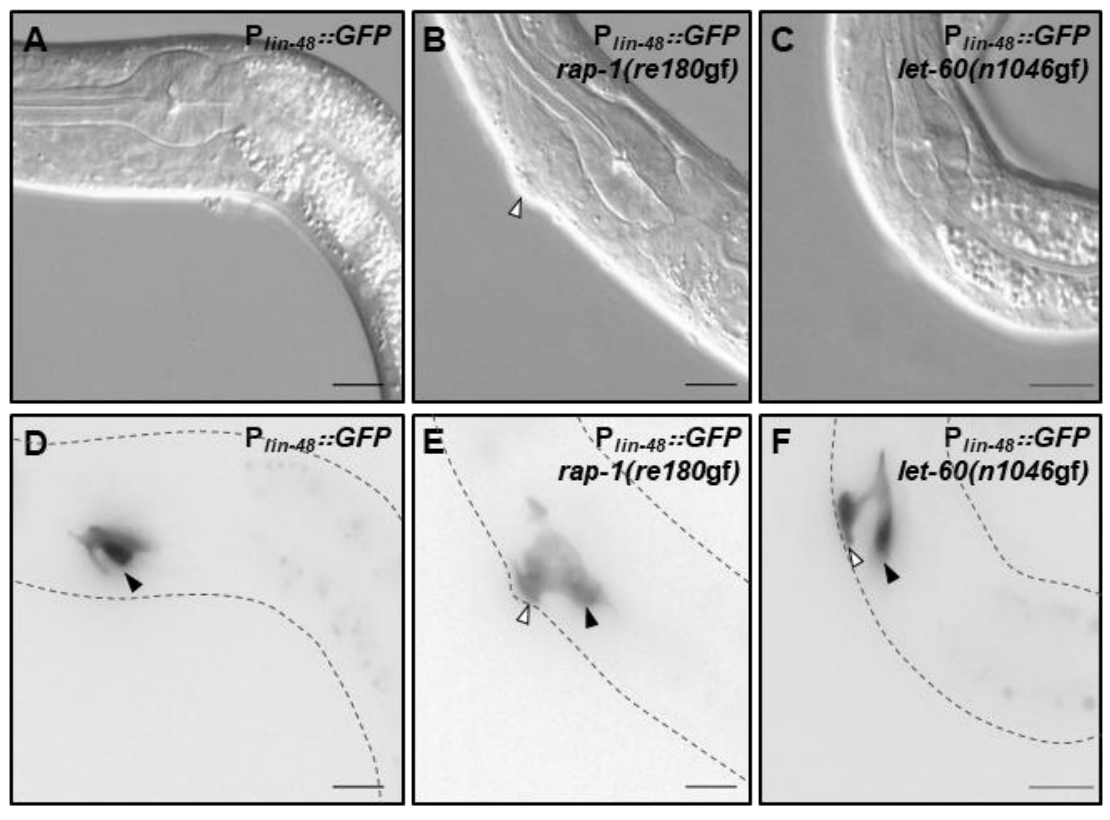

G

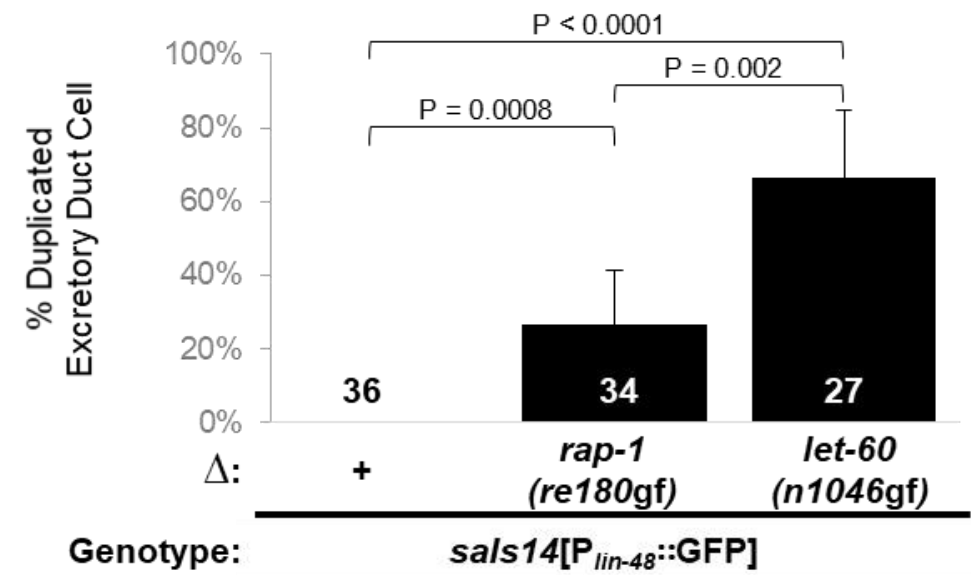

489 Figure 6. RAP-1 is sufficient to duplicate excretory duct cells.

Representative DIC (A, B,C) and inverted epifluorescence (D,E,F) micrographs of excretory duct cell fate marker sals 14[Plin-48::GFP] at the $\mathrm{L} 1$ stage. Wild-type animals $(\mathbf{A}, \mathbf{D})$ have a single duct, but both rap-1(re180gf) (B, E) and let-60(n1046gf) (C, F) are sufficient to duplicate the excretory duct. Scale bar $=10 \mu \mathrm{m}$. G) Counting animals with two cells positive for $\mathrm{P}_{\text {lin-48 }}:$ GFP expression indicated that both activated rap-1 and let-60 resulted in significantly increased duct cell duplications compared to the wild type. Numbers in columns $=\mathrm{N}$. Error bars $=95 \%$ confidence interval based on sample size, $P$ values were calculated by Fishers Exact -test. 
A
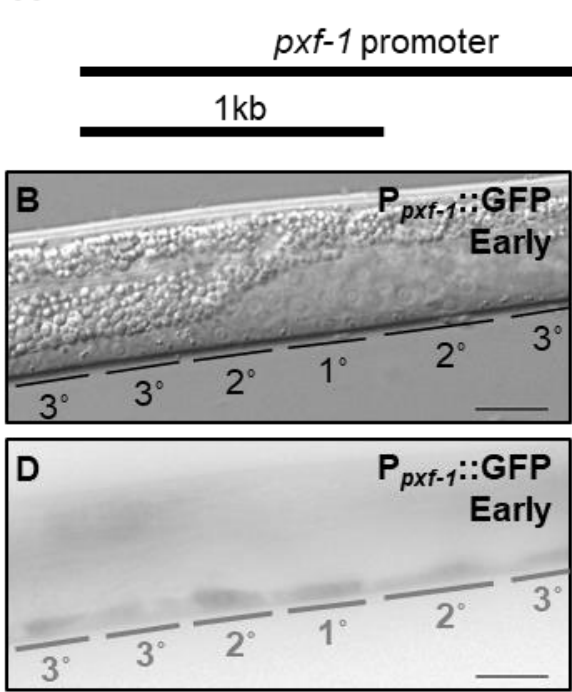
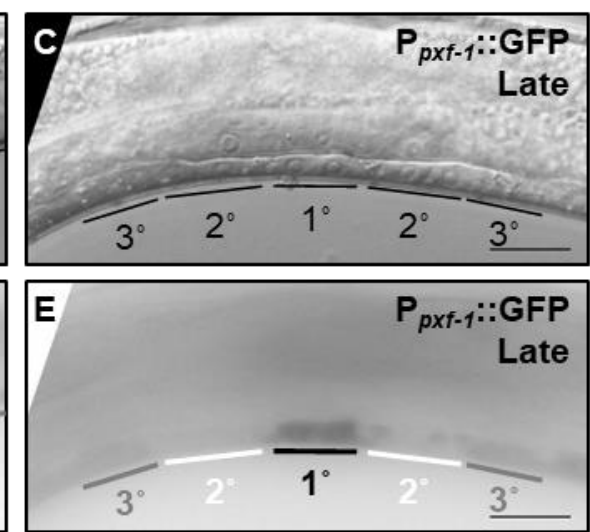

\section{GFP}

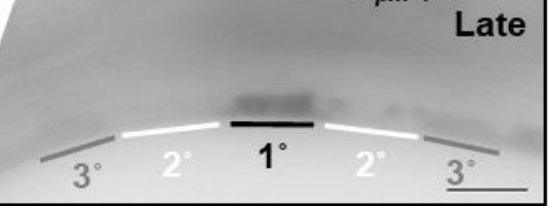

$\mathbf{F}$

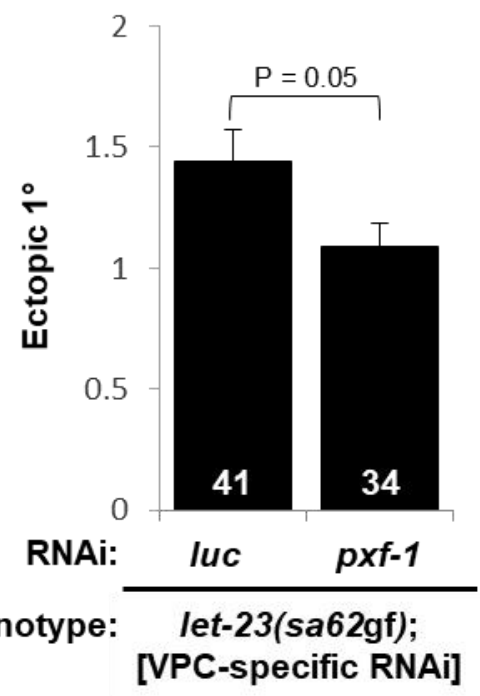

Figure 7. PXF-1/RapGEF contributes to $1^{\circ} \mathrm{VPC}$ induction.

A) Diagram of the $P_{p x f-1}:$ GFP reporter construct. Representative DIC micrographs of L3 larvae at the B) early 1-cell stage, prior to EGF signaling, and the C) 2-cell stage, after EGF signaling. D, E) Representative inverted epifluorescence micrographs of the animals shown in A, B), showing GFP expression from a pxf-1 transcriptional reporter GFP fusion transgene (bj/s4O[P $\left.\left.P_{p x-1}:: G F P\right]\right)$. D) GFP was observed throughout the VPCs (gray labels) prior to EGF induction at the early 1-cell stage. E) At the 2-cell stage, GFP increased in presumptive $1^{\circ}$ (black labels) and decreased in presumptive $2^{\circ}$ cells (white labels); expression in presumptive $3^{\circ} \mathrm{s}$ did not change, gray labels. (scale bar $=10 \mu \mathrm{m}$ ). F) VPC-specific RNAi knockdown of pxf-1 in the let23(sa62gf) background decreased ectopic $1^{\circ}$ induction compared to Luciferase (luc) RNAi control (sa62gf

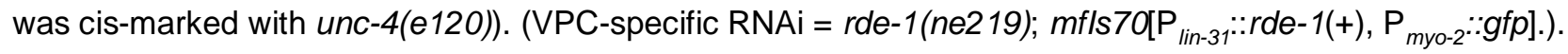
The $\mathrm{Y}$-axis indicates number of ectopic $1^{\circ} \mathrm{s}$. All data are representative non-pooled assays. Error bars = S.E.M., white numbers $=\mathrm{N}$. $\mathrm{P}$ values were calculated by T-test. 


\begin{tabular}{|c|c|c|}
\hline & Effector Binding & \\
\hline $\mathrm{N}-$ Ras & QNHFVDEYDPTIEDSYRKQVVIDGE & \\
\hline H-Ras & QNHFVDEYDPTIEDSYRKQVVIDGE & Ras \\
\hline K-Ras & QNHFVDEYDPTIEDSYRKQVVIDGE & Ino. \\
\hline LET -60 & QNHFVEEYDPTIEDSYRKQVVIDGE & \\
\hline Rap1A & QGIFVEKYDPTIEDSYRKQVEVDCQ & \\
\hline Rap1B & QGIFVEKYDPTIEDSYRKQVEVDAQ & Rap \\
\hline RAP-1 & QGIFVEKYDPTIEDSYRKQVEVDGQ & \\
\hline $\operatorname{Rap} 2 \mathrm{~A}$ & TGTFIEKYDPTIEDFYRKEIEVDSS & \\
\hline Rap2B & TGSFIEKYDPTIEDFYRKEIEVDSS & \\
\hline Rap2C & TGTFIEKYDPTIEDFYRKEIEVDSS & \\
\hline RAP-2 & SSTFIEKYDPTIEDEYRKEIEVDGQ & \\
\hline RAP -3 & QGIFVHTYDATIEDSYRKLSKVDAE & $\mathbf{R}$ \\
\hline
\end{tabular}

493 Figure S1. Only Rap1 shares complete Effector Binding Domain conservation with Ras

A) Sequence alignment of the effector binding domain of Ras, Rap1, Rap2, and Rap3 family members. The core effector domain is shaded in red and identical residues, yellow. C. elegans genes are shaded in blue and human genes not highlighted. 

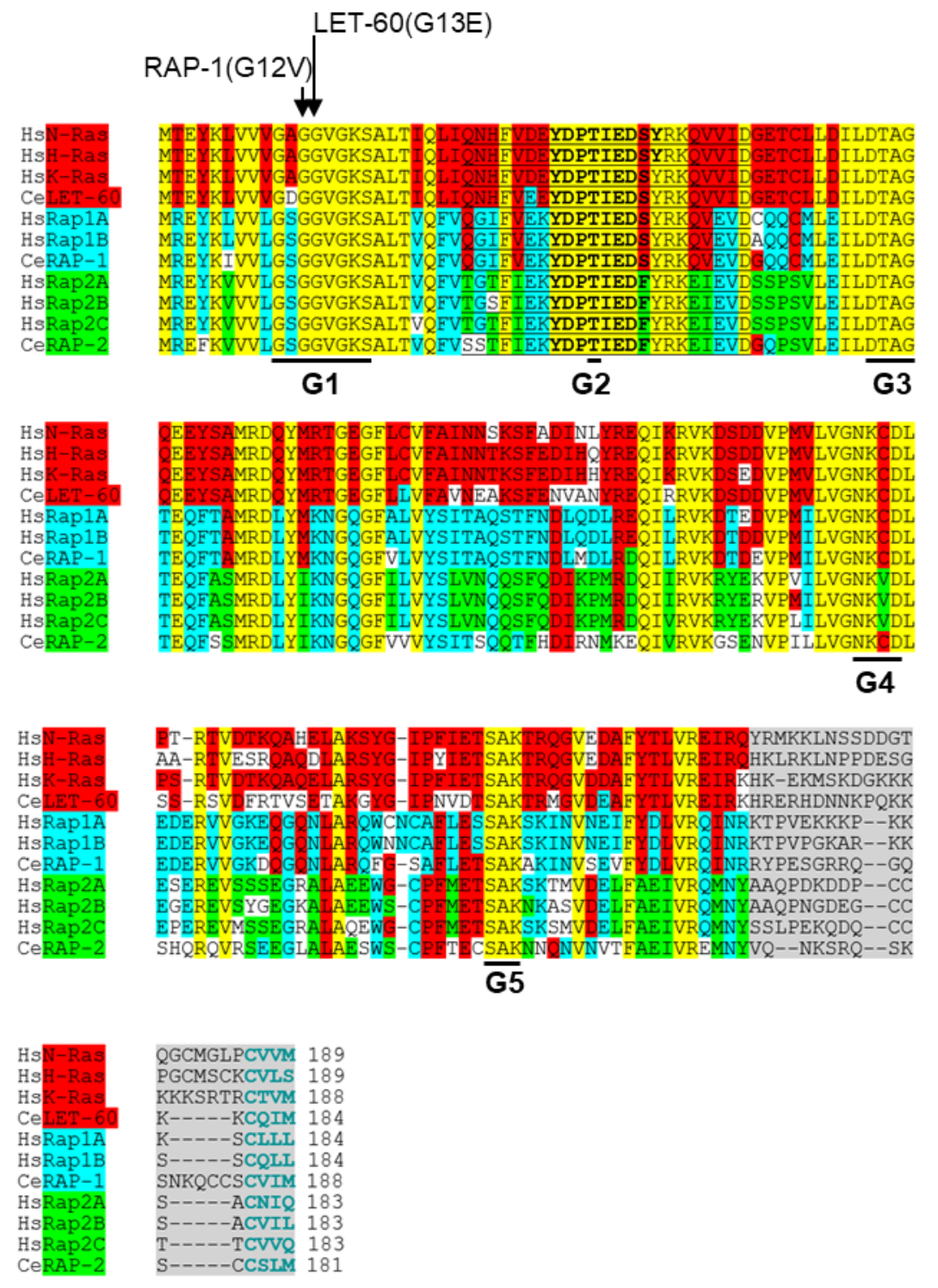

495 Figure S2. Rap1 shares greater homology with Ras than Rap2

A) Sequence alignment of human ( $\mathrm{Hs})$ and $C$. elegans $(\mathrm{Ce})$ orthologs of Ras, Rap1, and Rap2. Identical residues are highlighted in yellow, with Rap1 and Rap2 conserved residues in blue and green, respectively. The effector domain of the Ras family members Is underlined with the core domain in bolded text. The C-terminal hypervariable region is highlighted in gray and the CAAX in blue text. 
A
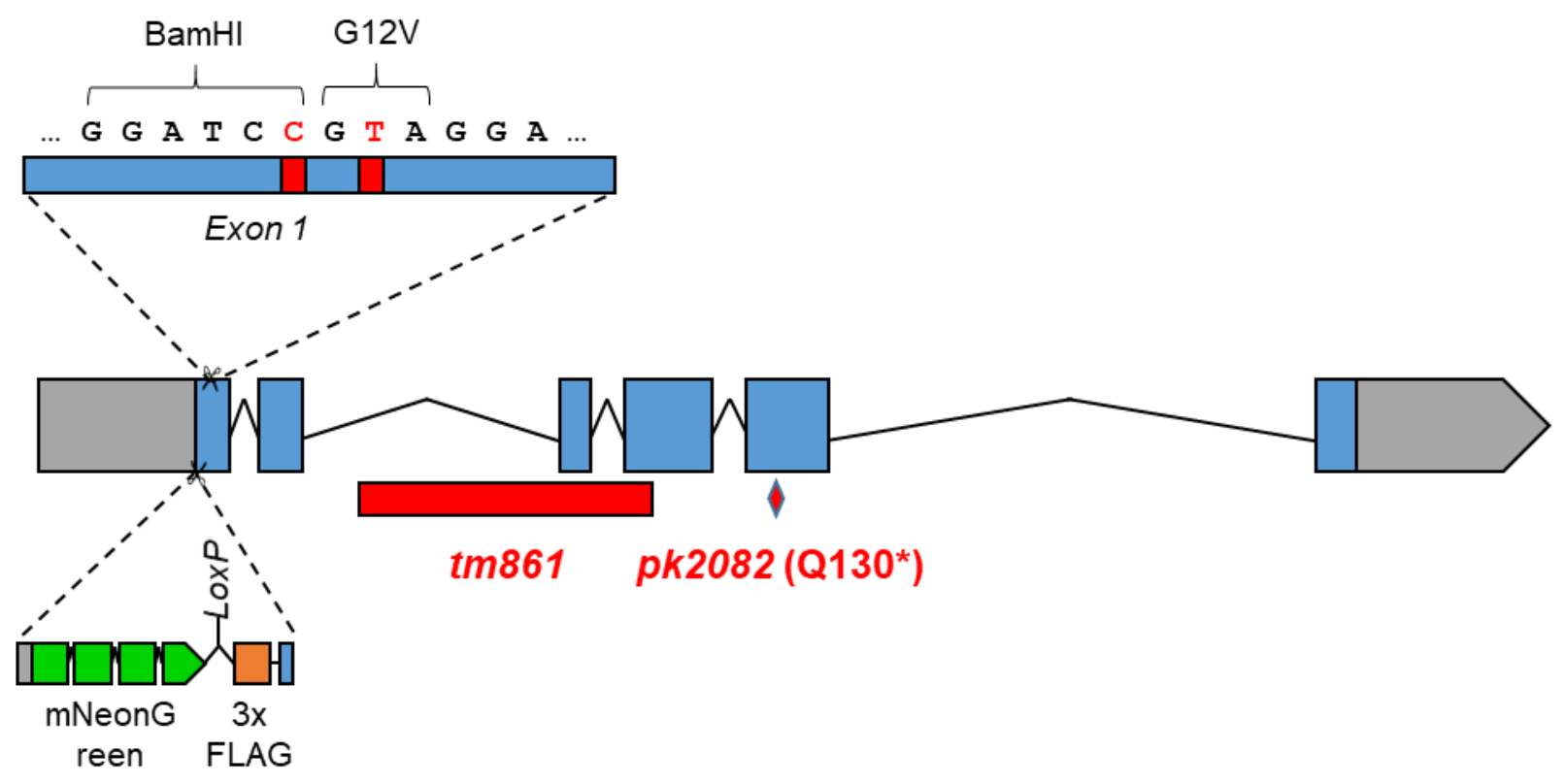

ср151

497 Figure S3. Overview of all rap-1 alleles used

A) Diagram of all the rap-1 alleles used in this study. CRISPR knock-ins are colored in green and predicted strong loss or null alleles in red. 

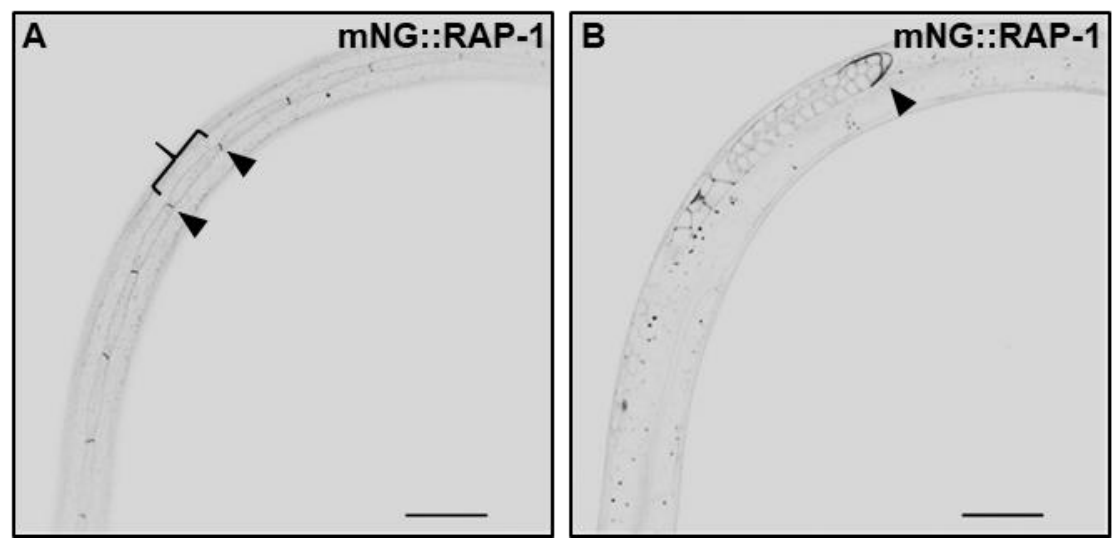

499 Figure S4. Endogenous tagged RAP-1 is ubiquitously expressed and localizes to plasma membranes and junctions.

A,B) Representative inverted confocal fluorescence micrographs of a single rap-1(cp151[mNG^3xFlag::rap-1]) animal at different focal planes shows expression in (A) hypodermal seam cells with localization at plasma membranes (bracket) and enrichment at the adherens junctions between cells (black arrowheads), (B) localization to plasma membranes in the germline, with increased expression in the migrating distal tip cell (black arrowhead) that invades through basement membranes. Scale bar $=20 \mu \mathrm{m}$. 

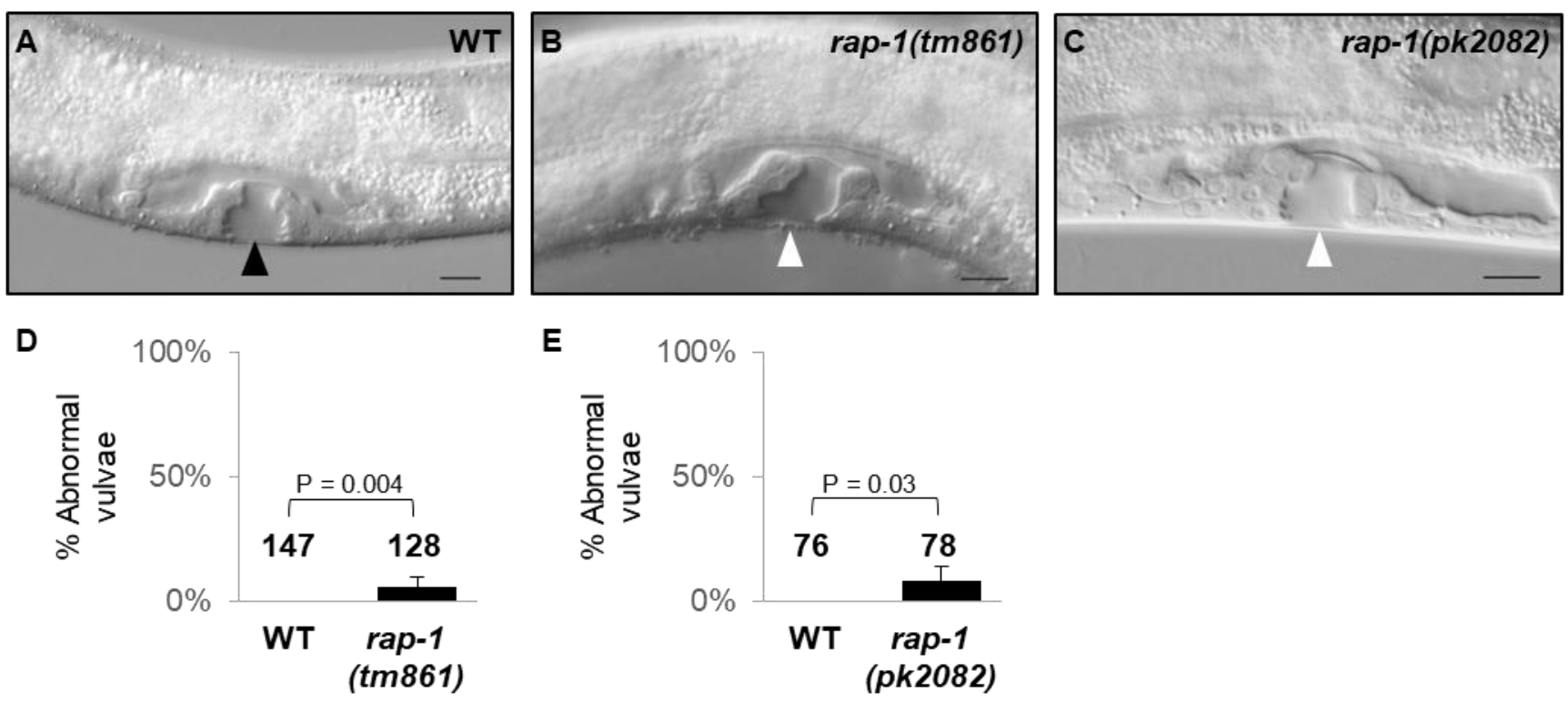

501 Figure S5. Loss of rap-1 confers low penetrance vulval patterning defects.

Representative DIC micrographs of vulvae at the late L4 stage. A) Wild-type vulva (black triangle). B) rap1(tm861) and C) rap-1(pk2082) abnormal vulvae (white triangle). Scale bar $=10 \mu \mathrm{m}$. D, E) Quantification of mutant abnormal vulvae in wildtype vs. (D) rap-1(tm861) and (E) rap-1(pk2082), with percent abnormal vulvae on the $\mathrm{Y}$ axis. All data are representative non-pooled assays with the mean shown. Numbers in columns $=\mathrm{N}$. Error bars $=95 \%$ confidence interval based on sample size, $P$ values were calculated by Fishers Exact-test. 


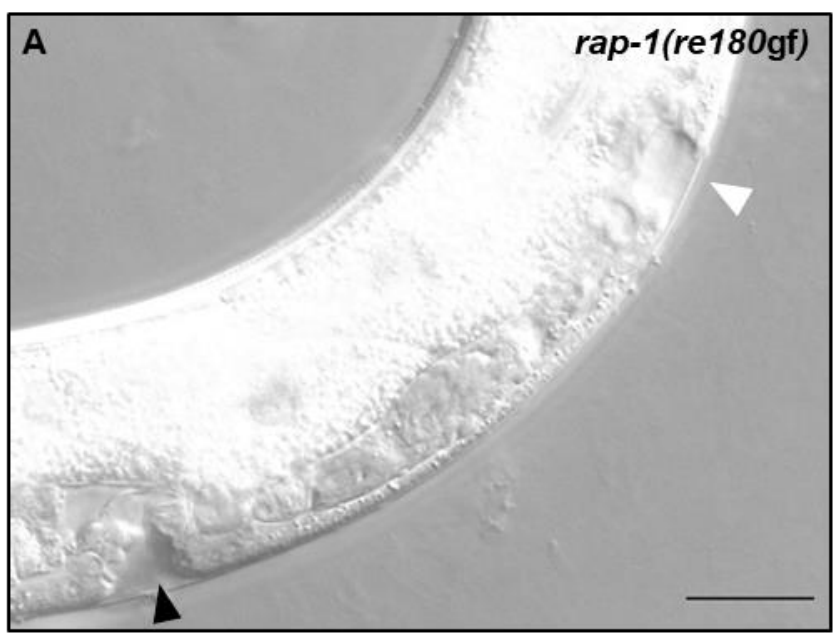

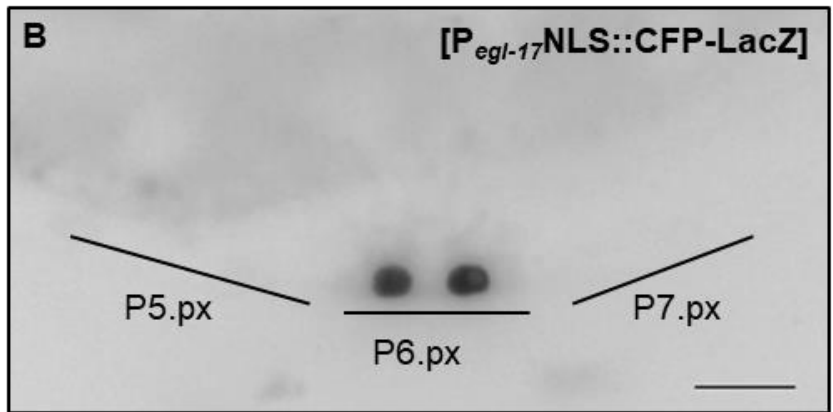

D

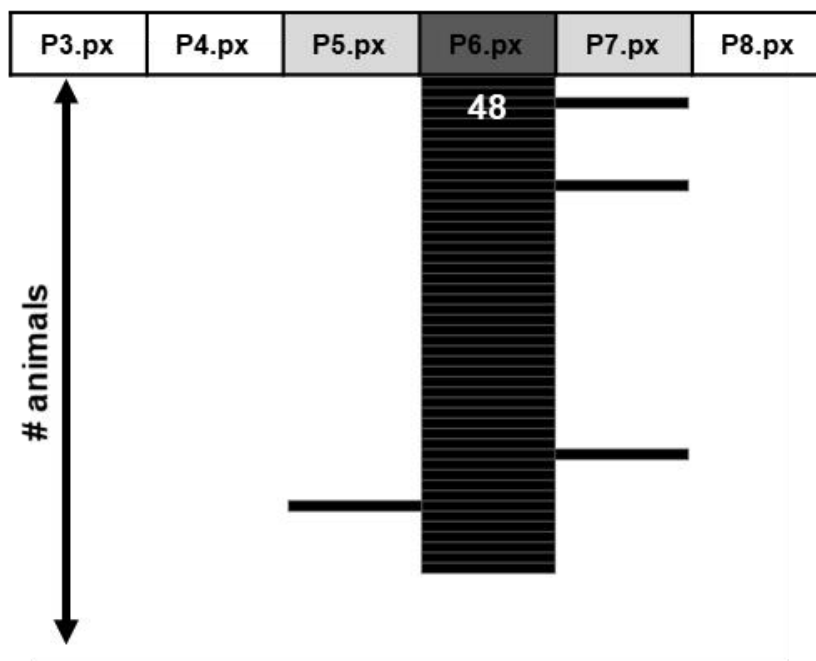

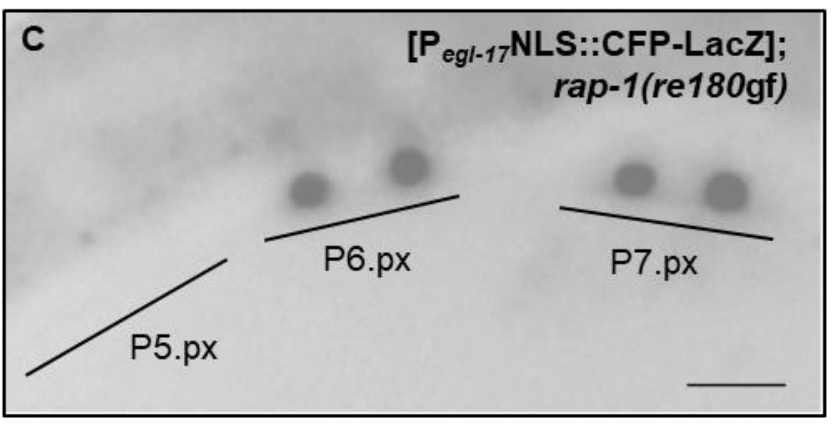

E

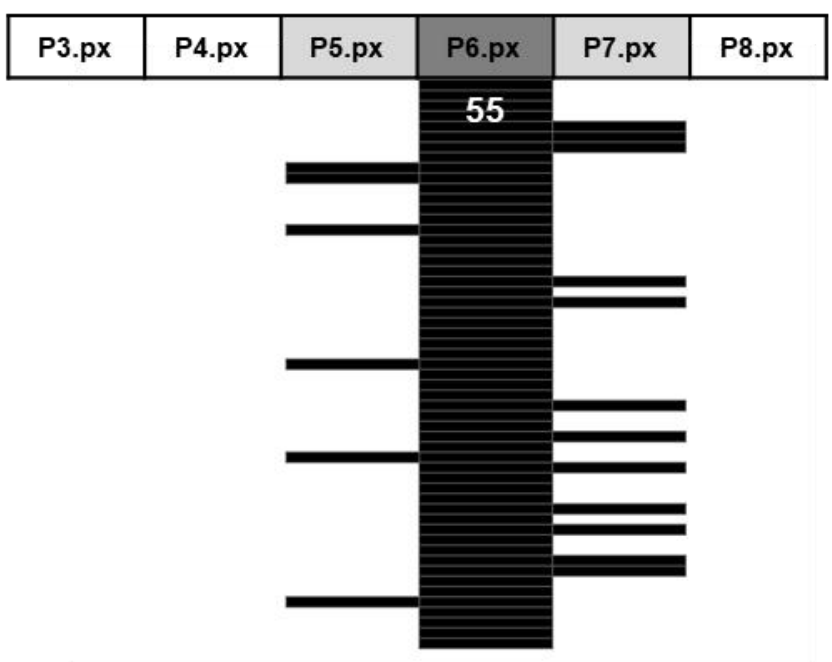

Figure S6. rap-1(re180gf) is sufficient to induce ectopic $1^{\circ} \mathrm{VPCs}$

A) Representative DIC micrographs at the late L4 stage shows the wild-type vulva (black triangle) and induction of ectopic $1^{\circ} \mathrm{s}$ (white triangle) in rap-1(re180gf) animals (scale bar $=100$ pixels). B, C) Representative epifluorescence micrographs in arls92 [ $\mathrm{P}_{\text {egl-1 }} \mathrm{NLS}$ ::CFP-LacZ] animals at the 2-cell (Pn.px) stage show the inappropriate expression of $1^{\circ}$ signaling reporter $\left[\mathrm{P}_{\text {egl- } 17} \mathrm{NLS}:\right.$ :CFP-LacZ] in (C) rap-1(re180gf) but not (B) wild

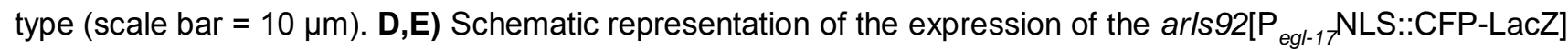
signaling reporter across the all six VPCs for both (D) control and (E) rap-1(re180gf). The addition of rap1(re180gf) resulted in a significant increase of exprgssion outside of P6.px ( $P=0.003) P$ values were calculated by Fisher's Exact Test. $\mathrm{N}=$ white number, with each line representing an animal. 
A

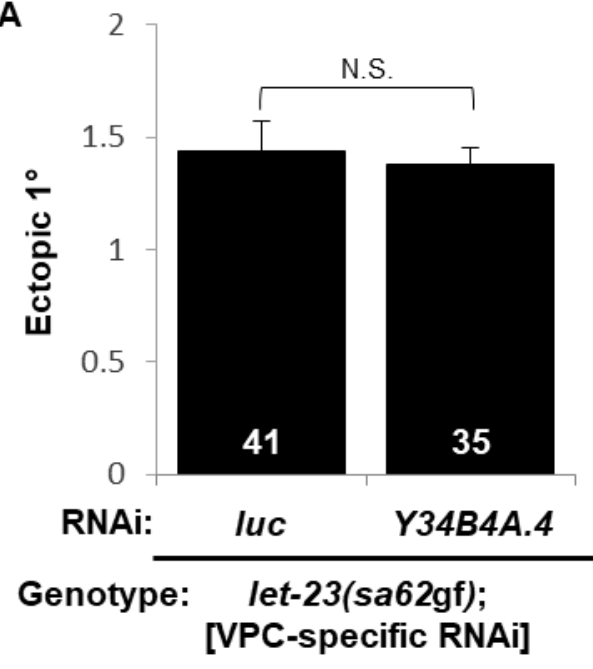

505 Figure S7. Y34B4A.4/Rap1GEF does not contribute to $1^{\circ}$ VPC induction.

A) VPC-specific RNAi knockdown of Y34B4A.4 (C. elegans ortholog of Rap1GEF) in the let-23(sa62gf) background had no effect compared to Luciferase (luc) RNAi control (sa62 was cis-marked with unc-4(e120)). (VPC-specific RNAi = rde-1(ne219); mfls70[P $\mathrm{P}_{\text {lin-31 }}: \because$ rde-1(+), $\mathrm{P}_{\text {myo-2 } 2: \because g f p] .)}$ The Yaxis indicates number of ectopic $1^{\circ} \mathrm{s}$. All data are representative non-pooled assays. Error bars $=$ S.E.M., white numbers $=\mathrm{N}$. $P$ values were calculated by T-test. 


\section{Supplementary Table 1}

\section{Strains}

\begin{tabular}{|c|c|c|}
\hline Strain & Genotype & Used in Figures \\
\hline LP399 & rap-1(cp151[mNeonGreen^3xFlag::rap-1]) IV & Fig. 2, S3 \\
\hline FZ222 & rap-1(tm861) IV & \\
\hline DV3152 & rap-1(tm861) IV (Outcrossed 12x) & Fig. S4 \\
\hline FZ181 & rap-1(pk2082) IV & Fig. S4 \\
\hline MT2124 & let-60(n1046gf) IV & Fig. 3 \\
\hline DV3512 & let-60(n1046gf) rap-1(tm861) IV & Fig. 3 \\
\hline PS1524 & $\begin{array}{l}\text { unc-4(e120) let-23(sa62gf)/mnC1 dpy-10(e128) unc- } \\
52(e 444) \text { II }\end{array}$ & Fig. 3 \\
\hline DV2901 & unc-4(e120) let-23(sa62gf) II; rap-1(tm861) IV & Fig. 3 \\
\hline DV2968 & $\begin{array}{l}\text { unc-4(e120) let-23(sa62gf) II; mfIs70[P lin-31::rde-1(+), } \\
\mathrm{P}_{\text {myo-2::gfp] IV; rde-1(ne219) V }}\end{array}$ & Fig. $3,7, S 7$ \\
\hline DV3313 & rap-1(re180gf) IV (Outcrossed 3x) & Fig. 4 \\
\hline DV2261 & $\begin{array}{l}\text { arls92[P egl-17::NLS-CFP-LacZ, P Pttx-3::gfp, unc-4(+)] } \\
\text { mrt-(re58) V }\end{array}$ & Fig. S5 \\
\hline DV3391 & $\begin{array}{l}\text { rap-1(re180gf) IV; arls92[P egl-17::NLS-cfp-LacZ, Pttx-3:: } \\
\text { gfp, unc-4(+)] mrt-(re58) V }\end{array}$ & Fig. S5 \\
\hline GS4892 & 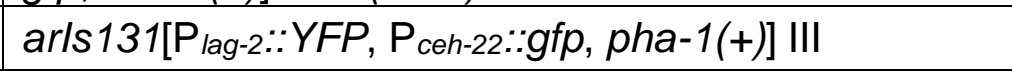 & Fig. 5 \\
\hline DV3392 & $\begin{array}{l}\text { arls131[Plag-2::YFP, Pceh-22::gfp, pha-1(+)] III; rap- } \\
\text { 1(re180gf) IV }\end{array}$ & Fig. 5 \\
\hline CM117 & unc-119(e2498) III; sals14[P lin-48:::gfp, unc-119(+)] & Fig. 6 \\
\hline DV2353 & $\begin{array}{l}\text { unc-119(e2498) III; sals14[P lin-48:::gfp, unc-119(+)];let- } \\
\text { 60(n1046gf) IV }\end{array}$ & Fig. 6 \\
\hline DV3393 & $\begin{array}{l}\text { unc-119(e2498) III; sals14[Plin-48::gfp, unc-119(+)]; } \\
\text { rap-1(re180gf) IV }\end{array}$ & Fig. 6 \\
\hline FZ117 & bjls40[P $\mathrm{P}_{p x f-1:: g f p]}$ & Fig. 7 \\
\hline CB2065 & $d p y-11(e 224)$ unc-76(e911) V & \\
\hline JK2958 & $n T 1[q / s 51](\mathrm{IV} ; \mathrm{V}) / d p y-11(\mathrm{e} 224)$ unc-42(e270) V & \\
\hline
\end{tabular}


509 Supplementary Table 2

510 RNAi

511

512

513

514

515

516

517

\begin{tabular}{|l|l|l|}
\hline RNAi target & $\begin{array}{l}\text { Source BioScience Library } \\
\text { Position }\end{array}$ & Used in Figures \\
\hline luciferase & pREW2 & Fig. 3, 4, 7, S7 \\
\hline rap-1 (sjj_C27B7.8) & IV-4F17 & Fig. 3 \\
\hline lip-1 (sjj_C05B10.1) & IV-3I16 & Fig. 3, 4 \\
\hline gap-1 (sjj_T24C12.2) & X-1D10 & Fig. 3 \\
\hline pxf-1 (sjj_T14G10.2) & IV-5I14 & Fig. 7 \\
\hline $\begin{array}{l}\text { "Rap1GEF"(sjj2_Y34B4A. } \\
\text { 4) }\end{array}$ & X-8D15 & Fig. S7 \\
\hline pop-1 (sjj_W10C8.2) & I-1K04 & Used as RNAi control \\
\hline
\end{tabular}

\section{Supplementary Table 3}

Primers

\begin{tabular}{|l|l|l|l|}
\hline Name & Sequence & $\mathbf{T}_{\mathbf{a}}$ & Used for \\
\hline oNR001 & TTCGTGCAGGGAATTTTCGT & $50^{\circ} \mathrm{C}$ & rap-1(tm861) genotyping \\
\hline oNR004 & GAGCAATTCACAGCGATGC & $50^{\circ} \mathrm{C}$ & rap-1(tm861) genotyping \\
\hline oNR003 & $\begin{array}{l}\text { TGTTAGCCTCCTTTTCATTGAG } \\
\text { A }\end{array}$ & $50^{\circ} \mathrm{C}$ & rap-1(tm861) genotyping \\
\hline oNR122 & $\begin{array}{l}\text { TGTGTCATCTGGTCTGTACTTG } \\
\text { G }\end{array}$ & $50^{\circ} \mathrm{C}$ & rap-1(re180gf) genotyping \\
\hline oNR123 & TCCCCTGCACGAATTGTACC & $50^{\circ} \mathrm{C}$ & rap-1(re180gf) genotyping \\
\hline oNR114 & $\begin{array}{l}\text { ATTGTTGTGCTCGGATCTGGG } \\
\text { TTTAAGAGCTATGCTGGAAACA } \\
\text { G }\end{array}$ & $53^{\circ} \mathrm{C}$ & $\begin{array}{l}\text { rap-1(G12V) } \\
\text { sgRNA mutagenesis - FW }\end{array}$ \\
\hline oNR024 & CAAGACATCTCGCAATAGG & $53^{\circ} \mathrm{C}$ & sgRNA mutagenesis - RV \\
\hline
\end{tabular}

\section{Supplementary Table 4}

\section{Plasmids}

\begin{tabular}{|l|l|l|}
\hline Name & Description & Used for \\
\hline pNR21 & $\begin{array}{l}\text { pJW1236 rap-1(G12V) sgRNA }+ \\
\text { Cas9 }\end{array}$ & rap-1(G12V) CRISPR knock-in \\
\hline pJA58 & $d p y-10($ cn64) sgRNA & $d p y-10(c n 64)$ co-CRISPR knock-in \\
\hline pPD118.33 & Pmyo-2::GFP & Co-injection marker \\
\hline
\end{tabular}


518 Supplementary Table 5

519 sgRNA sequences and PAMs

\begin{tabular}{|l|l|}
\hline sgRNA sequence and PAM & Used for \\
\hline ATTGTTGTGCTCGGATCTGGAGG & rap-1 \\
\hline GCTACCATAGGCACCACGAGEG & $d p y-10$ \\
\hline
\end{tabular}

\section{Supplementary Table 6}

Repair SsODN

\begin{tabular}{|c|c|}
\hline ssODN sequence & Description \\
\hline $\begin{array}{l}\text { 5'- } \\
\text { GAATGCGGGAGTATAAGATTGTTGTGCTCGGATCCGT } \\
\text { AGGAGTAGGAAAATCCGCACTGGTATAG- 3' }\end{array}$ & $\begin{array}{l}\text { Repair ssODN for rap- } \\
1(\mathrm{G} 12 \mathrm{~V})(65 \mathrm{bp})\end{array}$ \\
\hline $\begin{array}{l}\text { 5'- } \\
\text { CACTTGAACTTCAATACGGCAAGATGAGAATGACTGG } \\
\text { AAACCGTACCGCATGCGGTGCCTATGGTAGCGGAGC } \\
\text { TTCACATGGCTTCAGACCAACAGCCTAT- 3' }\end{array}$ & $\begin{array}{l}\text { Repair ssODN for } d p y- \\
10(c n 64)(101 \mathrm{bp})\end{array}$ \\
\hline
\end{tabular}




\section{REFERENCES}

526 Altschuler, D.L., and F. Ribeiro-Neto, 1998 Mitogenic and oncogenic properties of the small G protein Rap1b. Proc Natl Acad Sci U S A 95 (13):7475-7479.

Aoki, Y., T. Niihori, S. Inoue, and Y. Matsubara, 2016 Recent advances in RASopathies. J Hum Genet 61 (1):33-39.

Baril, C., M. Lefrancois, M. Sahmi, H. Knaevelsrud, and M. Therrien, 2014 Apical accumulation of the Sevenless receptor tyrosine kinase during Drosophila eye development is promoted

Barkoulas, M., J.S. van Zon, J. Milloz, A. van Oudenaarden, and M.A. Felix, 2013 Robustness and epistasis in the C. elegans vulval signaling network revealed by pathway dosage modulation. Dev Cell 24 (1):64-75.

Beitel, G.J., S.G. Clark, and H.R. Horvitz, 1990 Caenorhabditis elegans ras gene let-60 acts as a switch in the pathway of vulval induction. Nature 348 (6301):503-509.

Berset, T., E.F. Hoier, G. Battu, S. Canevascini, and A. Hajnal, 2001 Notch inhibition of RAS signaling through MAP kinase phosphatase LIP-1 during C. elegans vulval development. Science 291 (5506):1055-1058.

Berset, T.A., E.F. Hoier, and A. Hajnal, 2005 The C. elegans homolog of the mammalian tumor suppressor Dep-1/Scc1 inhibits EGFR signaling to regulate binary cell fate decisions.

544 Bogershausen, N., I.C. Tsai, E. Pohl, P.O. Kiper, F. Beleggia et al., 2015 RAP1-mediated MEK/ERK pathway defects in Kabuki syndrome. J Clin Invest 125 (9):3585-3599. 
547 Braendle, C., and M.A. Felix, 2008 Plasticity and errors of a robust developmental system in different environments. Dev Cell 15 (5):714-724.

Brenner, S., 1974 The genetics of Caenorhabditis elegans. Genetics 77 (1):71-94.

550 Caron, E., A.J. Self, and A. Hall, 2000 The GTPase Rap1 controls functional activation of macrophage integrin alphaMbeta2 by LPS and other inflammatory mediators. Curr Biol $10(16): 974-978$.

553 Chang, C., N.A. Hopper, and P.W. Sternberg, 2000 Caenorhabditis elegans SOS-1 is necessary for multiple RAS-mediated developmental signals. Embo j 19 (13):3283-3294.

555 Chen, N., and I. Greenwald, 2004 The lateral signal for LIN-12/Notch in C. elegans vulval development comprises redundant secreted and transmembrane DSL proteins. Dev Cell 6

Cook, S.J., B. Rubinfeld, I. Albert, and F. McCormick, 1993 RapV12 antagonizes Ras-dependent activation of ERK1 and ERK2 by LPA and EGF in Rat-1 fibroblasts. Embo $j 12$

Dent, J.A., and M. Han, 1998 Post-embryonic expression pattern of C. elegans let-60 ras reporter (9):3475-3485. constructs. Mech Dev 72 (1-2):179-182.

Dickinson, D.J., A.M. Pani, J.K. Heppert, C.D. Higgins, and B. Goldstein, 2015 Streamlined Genome Engineering with a Self-Excising Drug Selection Cassette. Genetics 200 ras homologue let-60 by a novel, temperature-sensitive, gain-of-function mutation. Genetics 146 (2):553-565. 
569 Frische, E.W., W. Pellis-van Berkel, G. van Haaften, E. Cuppen, R.H. Plasterk et al., 2007 RAP1 and the RAL-1/exocyst pathway coordinate hypodermal cell organization in Caenorhabditis elegans. Embo j 26 (24):5083-5092.

572 Gloerich, M., and J.L. Bos, 2011 Regulating Rap small G-proteins in time and space. Trends Cell Biol 21 (10):615-623.

574 Greenwald, I., and R. Kovall, 2013 Notch signaling: genetics and structure. WormBook:1-28.

575 Greenwald, I.S., P.W. Sternberg, and H.R. Horvitz, 1983 The lin-12 locus specifies cell fates in Caenorhabditis elegans. Cell 34 (2):435-444.

577 Grewal, T., M. Koese, F. Tebar, and C. Enrich, 2011 Differential Regulation of RasGAPs in Cancer. Genes Cancer 2 (3):288-297.

579 Guo, X.X., S. An, Y. Yang, Y. Liu, Q. Hao et al., 2016 Rap-Interacting Proteins are Key Players in the Rap Symphony Orchestra. Cell Physiol Biochem 39 (1):137-156.

581 Gyan, E., M. Frew, D. Bowen, C. Beldjord, C. Preudhomme et al., 2005 Mutation in RAP1 is a rare event in myelodysplastic syndromes. Leukemia 19 (9):1678-1680.

Hagedorn, E.J., H. Yashiro, J.W. Ziel, S. Ihara, Z. Wang et al., 2009 Integrin acts upstream of netrin signaling to regulate formation of the anchor cell's invasive membrane in $\mathrm{C}$. elegans. Dev Cell 17 (2):187-198.

Hajnal, A., C.W. Whitfield, and S.K. Kim, 1997 Inhibition of Caenorhabditis elegans vulval induction by gap-1 and by let-23 receptor tyrosine kinase. Genes Dev 11 (20):2715-2728.

Han, M., R.V. Aroian, and P.W. Sternberg, 1990 The let-60 locus controls the switch between vulval and nonvulval cell fates in Caenorhabditis elegans. Genetics 126 (4):899-913. vulval induction, encodes a ras protein. Cell 63 (5):921-931. 
Hancock, J.F., H. Paterson, and C.J. Marshall, 1990 A polybasic domain or palmitoylation is required in addition to the CAAX motif to localize p21ras to the plasma membrane. Cell 63 (1):133-139.

Hara, M., and M. Han, 1995 Ras farnesyltransferase inhibitors suppress the phenotype resulting from an activated ras mutation in Caenorhabditis elegans. Proc Natl Acad Sci U S A 92 (8):3333-3337.

Hariharan, I.K., R.W. Carthew, and G.M. Rubin, 1991 The Drosophila roughened mutation: activation of a rap homolog disrupts eye development and interferes with cell

Johnson, A.D., D. Fitzsimmons, J. Hagman, and H.M. Chamberlin, 2001 EGL-38 Pax regulates the ovo-related gene lin-48 during Caenorhabditis elegans organ development. Methods 30 (4):313-321.

Katz, W.S., G.M. Lesa, D. Yannoukakos, T.R. Clandinin, J. Schlessinger et al., 1996 A point mutation in the extracellular domain activates LET-23, the Caenorhabditis elegans epidermal growth factor receptor homolog. Mol Cell Biol 16 (2):529-537.

609 Kitayama, H., T. Matsuzaki, Y. Ikawa, and M. Noda, 1990 A domain responsible for the transformation suppressor activity in Krev-1 protein. Jpn J Cancer Res 81 (5):445-448.

611 Kitayama, H., Y. Sugimoto, T. Matsuzaki, Y. Ikawa, and M. Noda, 1989 A ras-related gene with transformation suppressor activity. Cell 56 (1):77-84.

613 Knox, A.L., and N.H. Brown, 2002 Rap1 GTPase regulation of adherens junction positioning and cell adhesion. Science 295 (5558):1285-1288. 
615 Krauthammer, M., Y. Kong, B.H. Ha, P. Evans, A. Bacchiocchi et al., 2012 Exome sequencing identifies recurrent somatic RAC1 mutations in melanoma. Nat Genet 44 (9):1006-1014.

617 Kupzig, S., D. Deaconescu, D. Bouyoucef, S.A. Walker, Q. Liu et al., 2006 GAP1 family members constitute bifunctional Ras and Rap GTPase-activating proteins. J Biol Chem

Lindsay, C.R., S. Lawn, A.D. Campbell, W.J. Faller, F. Rambow et al., 2011 P-Rex1 is required for efficient melanoblast migration and melanoma metastasis. Nat Commun 2:555.

Maertens, O., and K. Cichowski, 2014 An expanding role for RAS GTPase activating proteins (RAS GAPs) in cancer. Adv Biol Regul 55:1-14.

624 Mavromatakis, Y.E., and A. Tomlinson, 2012 The role of the small GTPase Rap in Drosophila R7 photoreceptor specification. Proc Natl Acad Sci U S A 109 (10):3844-3849.

McLaughlin, S.K., S.N. Olsen, B. Dake, T. De Raedt, E. Lim et al., 2013 The RasGAP gene, RASAL2, is a tumor and metastasis suppressor. Cancer Cell 24 (3):365-378.

Mishra, S., S.M. Smolik, M.A. Forte, and P.J. Stork, 2005 Ras-independent activation of ERK signaling via the torso receptor tyrosine kinase is mediated by Rap1. Curr Biol 15 (4):366-370.

635 O'Keefe, D.D., E. Gonzalez-Nino, M. Burnett, L. Dylla, S.M. Lambeth et al., 2009 Rap1 
638 Paix, A., Y. Wang, H.E. Smith, C.Y. Lee, D. Calidas et al., 2014 Scalable and versatile genome editing using linear DNAs with microhomology to Cas9 Sites in Caenorhabditis elegans. Genetics 198 (4):1347-1356.

641 Papke, B., and C.J. Der, 2017 Drugging RAS: Know the enemy. Science 355 (6330):1158-1163.

642 Pellis-van Berkel, W., M.H. Verheijen, E. Cuppen, M. Asahina, J. de Rooij et al., 2005

643 Requirement of the Caenorhabditis elegans RapGEF pxf-1 and rap-1 for epithelial integrity. Mol Biol Cell 16 (1):106-116.

645 Pizon, V., M. Desjardins, C. Bucci, R.G. Parton, and M. Zerial, 1994 Association of Rap1a and Rap1b proteins with late endocytic/phagocytic compartments and Rap2a with the Golgi

648 Prior, I.A., and J.F. Hancock, 2012 Ras trafficking, localization and compartmentalized signalling. Semin Cell Dev Biol 23 (2):145-153.

650 Raaijmakers, J.H., and J.L. Bos, 2009 Specificity in Ras and Rap signaling. J Biol Chem 284 (17):10995-10999.

652 Reedquist, K.A., E. Ross, E.A. Koop, R.M. Wolthuis, F.J. Zwartkruis et al., 2000 The small GTPase, Rap1, mediates CD31-induced integrin adhesion. J Cell Biol 148 (6):1151-1158.

654 Reiner, D.J., 2011 Ras effector switching as a developmental strategy. Small GTPases 2 (2):109112.

656 Reiner, D.J., and E.A. Lundquist, 2016 Small GTPases. WormBook:1-99.

657 Sakoda, T., K. Kaibuchi, K. Kishi, S. Kishida, K. Doi et al., 1992 smg/rap1/Krev-1 p21s inhibit 658 the signal pathway to the c-fos promoter/enhancer from c-Ki-ras p21 but not from c-raf-1 kinase in NIH3T3 cells. Oncogene 7 (9):1705-1711. 
Schutzman, J.L., C.Z. Borland, J.C. Newman, M.K. Robinson, M. Kokel et al., 2001 The

Caenorhabditis elegans EGL-15 signaling pathway implicates a DOS-like multisubstrate adaptor protein in fibroblast growth factor signal transduction. Mol Cell Biol 21

Sergent, J., 1990 Furtive incursions into bicameral minds. Integrative and coordinating role of subcortical structures. Brain 113 ( Pt 2):537-568.

Shaye, D.D., and I. Greenwald, 2002 Endocytosis-mediated downregulation of LIN-12/Notch upon Ras activation in Caenorhabditis elegans. Nature 420 (6916):686-690.

Shaye, D.D., and I. Greenwald, 2005 LIN-12/Notch trafficking and regulation of DSL ligand activity during vulval induction in Caenorhabditis elegans. Development 132 (22):5081-

Sherwood, D.R., and P.W. Sternberg, 2003 Anchor cell invasion into the vulval epithelium in C. elegans. Dev Cell 5 (1):21-31.

Srijakotre, N., J. Man, L.M. Ooms, C.M. Lucato, A.M. Ellisdon et al., 2017 P-Rex1 and P-Rex2 RacGEFs and cancer. Biochem Soc Trans 45 (4):963-977.

675 Sternberg, P.W., 2005 Vulval development. WormBook:1-28.

676 Stetak, A., P. Gutierrez, and A. Hajnal, 2008 Tissue-specific functions of the Caenorhabditis elegans p120 Ras GTPase activating protein GAP-3. Dev Biol 323 (2):166-176.

678 Sundaram, M.V., 2013 Canonical RTK-Ras-ERK signaling and related alternative pathways. WormBook:1-38.

680 Vossler, M.R., H. Yao, R.D. York, M.G. Pan, C.S. Rim et al., 1997 cAMP activates MAP kinase and Elk-1 through a B-Raf- and Rap1-dependent pathway. Cell 89 (1):73-82. 
682 Wang, M., and P.W. Sternberg, 1999 Competence and commitment of Caenorhabditis elegans vulval precursor cells. Dev Biol 212 (1):12-24.

684 Wennerberg, K., K.L. Rossman, and C.J. Der, 2005 The Ras superfamily at a glance. J Cell Sci 118 (Pt 5):843-846.

Yochem, J., M. Sundaram, and M. Han, 1997 Ras is required for a limited number of cell fates and not for general proliferation in Caenorhabditis elegans. Mol Cell Biol 17 (5):2716-

Yoo, A.S., C. Bais, and I. Greenwald, 2004 Crosstalk between the EGFR and LIN-12/Notch pathways in C. elegans vulval development. Science 303 (5658):663-666.

691 Yoo, A.S., and I. Greenwald, 2005 LIN-12/Notch activation leads to microRNA-mediated downregulation of Vav in C. elegans. Science 310 (5752):1330-1333.

693 York, R.D., H. Yao, T. Dillon, C.L. Ellig, S.P. Eckert et al., 1998 Rap1 mediates sustained MAP kinase activation induced by nerve growth factor. Nature 392 (6676):622-626.

Zand, T.P., D.J. Reiner, and C.J. Der, 2011 Ras effector switching promotes divergent cell fates in C. elegans vulval patterning. Dev Cell 20 (1):84-96.

697 Zhang, X., and I. Greenwald, 2011 Spatial regulation of lag-2 transcription during vulval precursor cell fate patterning in Caenorhabditis elegans. Genetics 188 (4):847-858.

699 Zhao, Z., C.C. Chen, C.D. Rillahan, R. Shen, T. Kitzing et al., 2015 Cooperative loss of RAS feedback regulation drives myeloid leukemogenesis. Nat Genet 47 (5):539-543.

701 Ziel, J.W., E.J. Hagedorn, A. Audhya, and D.R. Sherwood, 2009 UNC-6 (netrin) orients the 702 invasive membrane of the anchor cell in C. elegans. Nat Cell Biol 11 (2):183-189. 
bioRxiv preprint doi: https://doi.org/10.1101/297812; this version posted April 9, 2018. The copyright holder for this preprint (which was not certified by peer review) is the author/funder, who has granted bioRxiv a license to display the preprint in perpetuity. It is made available under aCC-BY-NC-ND 4.0 International license.

703 Zwartkruis, F.J., R.M. Wolthuis, N.M. Nabben, B. Franke, and J.L. Bos, 1998 Extracellular

704 signal-regulated activation of Rap1 fails to interfere in Ras effector signalling. Embo j 17

$705 \quad$ (20):5905-5912.

706 